\title{
Fonctions et usages de la représentativité patronale
}

Functions and uses of the representativeness of employers' organisations

\section{Nicole Maggi-Germain}

\section{(2) OpenEdition}

\section{Journals}

Édition électronique

URL : https://journals.openedition.org/travailemploi/5734

DOI : 10.4000/travailemploi.5734

ISSN : 1775-416X

Éditeur

DARES - Ministère du Travail

Édition imprimée

Date de publication : 15 septembre 2012

Pagination : 23-45

ISSN : 0224-4365

Référence électronique

Nicole Maggi-Germain, « Fonctions et usages de la représentativité patronale », Travail et Emploi [En ligne], 131 | 2012, mis en ligne le 01 décembre 2014, consulté le 21 septembre 2021. URL : http:// journals.openedition.org/travailemploi/5734; DOI : https://doi.org/10.4000/travailemploi.5734 


\title{
Fonctions et usages de la représentativité patronale
}

\author{
Nicole Maggi-Germain $\left.{ }^{*}\right)$
}

\begin{abstract}
D’un point de vue historique, la représentativité en tant que catégorie juridique a été pensée afin de permettre à des organisations professionnelles, tant patronales que de salariés, de signer des accords susceptibles d'extension, c'est-à-dire engageant l'ensemble des employeurs ou l'ensemble des salariés compris dans le champ d'application de la convention ou de l'accord. La reconnaissance de représentativité confère une telle légitimité. Cependant, les fonctions (les missions imparties par le droit), mais aussi les usages (l'utilisation qui en est faite par les organisations ou l'Administration) de la représentativité se sont progressivement élargis : le droit pose parfois la représentativité comme condition pour siéger dans des instances décisionnaires de la branche, dans des instances publiques consultatives ou encore pour participer au paritarisme national et interprofessionnel. En effet, si la représentativité constitue d'abord une forme de reconnaissance vis-à-vis de la profession, elle l'est aussi à l'égard de l'Administration : de facto ou de jure, elle confère une autorité pour peser sur les décisions publiques. De la même manière, l'Administration utilise la représentativité comme un critère de sélection des organisations professionnelles d'employeurs appelées à participer au dialogue social.
\end{abstract}

Si l'expression «organisations représentatives» est empruntée au Traité de Versailles signé le 28 juin $1919^{(1)}$, la représentativité en tant que catégorie juridique utilisée dans le champ des relations du travail date de 1936. D'un point de vue historique, elle a été pensée pour permettre à des organisations professionnelles, tant patronales qu'ouvrières, de signer des accords susceptibles d'extension c'està-dire applicables à des entreprises qui ne sont ni signataires ni adhérentes à l'organisation syndicale signataire. Mais cet engagement ne peut pas être le fait de n'importe quelle organisation, tant patronale que de salariés. Il faut qu'elle ait une légitimité lui permettant d'engager l'ensemble des employeurs ou l'ensemble des salariés. Prévue par le Code du travail, la représentativité des organisations professionnelles d'employeurs constitue une condition de validité de l'extension des conventions et accords collectifs de travail (art. L. 2261-19 cod. trav.) qui les transforme en un "contrat réglementaire» dont l'objet est de réglementer, d'une façon générale et uniforme, le contenu des contrats individuels de travail conclus ultérieurement. L'extension crée une situation juridique permanente, objective, impersonnelle qui fonde le «statut du travail» applicable

(*) Université de Paris 1, Panthéon-Sorbonne (ISST) Laboratoire Droit et changement social (UMR CNRS 6297), Maison des sciences de l'Homme Ange Guépin, Nantes; nicole. maggi-germain.nicole@univ-paris1.fr

(1) Il s'agit de permettre aux États membres de la Société des nations $(\mathrm{SDN})$ de désigner des représentants à la Conférence générale des représentants de l'Organisation internationale du travail. aux travailleurs (2). Il incombe aux services centraux du ministère du Travail, le cas échéant sous le contrôle du Conseil d'État en cas de contestation de la validité de l'arrêté d'extension, de vérifier, dans le cadre des enquêtes de représentativité prévues aux articles L. 2121-2(3) et R. 2121-1(4) du Code $\mathrm{du}$ travail, si cette condition est remplie. Plusieurs critères énoncés à l'article L. 2121-1, dans sa rédaction antérieure à la loi du 20 août $2008^{(5)}$, doivent être remplis : les effectifs, l'indépendance, les cotisations, l'expérience ou l'ancienneté du syndicat, l'attitude patriotique pendant l'Occupation. Son champ d'application n'étant pas limité aux seuls syndicats de salariés, l'Administration les a utilisés, s'agissant de la détermination de la représentativité des organisations professionnelles d'employeurs, de façon pragmatique ${ }^{(6)}$, faisant prévaloir les effec-

(2) Ce qui se traduit, par ailleurs, par un maintien des avantages individuels acquis en cas de dénonciation d'une convention ou d'un accord, étendus ou pas, non remplacés (art. L. 2261-13). (3) «S'il y a lieu de déterminer la représentativité d'un syndicat ou d'une organisation professionnelle autre que ceux affiliés à l'une des organisations représentatives au niveau national, l'autorité administrative diligente une enquête. L'organisation intéressée fournit les éléments d'appréciation dont elle dispose.»

(4) «Les enquêtes relatives à la détermination de la représentativité sont diligentées par le ministre chargé du travail. Pour les professions agricoles, ces attributions sont exercées en accord avec celui-ci par le ministre chargé de l'agriculture.» (5) Loi $n^{\circ} 2008-789$ portant rénovation de la démocratie sociale et réforme du temps de travail du 20 août 2008, Jo du 21 août, p. 13 064. Sur cette loi, voir, parmi d'autres, le dossier de la revue Droit social, «La rénovation de la démocratie sociale», $\mathrm{n}^{\circ}$ 6, juin 2009. Également BORENFREUND (2008).

(6) Cf. l'article de J.-P. Le Crom dans ce même numéro. 
tifs syndiqués tout en tenant compte d'un certain équilibre des relations sociales. C'est donc par un raisonnement analogique que l'Administration applique l'article L. 2121-1 aux organisations d'employeurs, tout en réalisant un important travail d'interprétation.

La loi du 20 août 2008 réforme en profondeur la représentativité des organisations syndicales, mettant un terme à la présomption irréfragable accordée aux seules organisations syndicales de salariés adhérant à la CGT, à la CFDT, à la CGT-FO, à la CFTC et à la CFE-CGC et intégrant l'élection comme l'un des critères de mesure de la représentativité. Il n'existe plus désormais qu'une représentativité prouvée. L'article L. 2121-1 reprend, en les modifiant et en les complétant, les critères cumulatifs de représentativité : respect des valeurs républicaines; indépendance; transparence financière; ancienneté minimale de deux ans dans le champ géographique et professionnel couvrant le niveau de négociation; audience; influence - prioritairement caractérisée par l'activité et l'expérience -; effectifs d'adhérents et cotisations. Le calcul de l'audience aux élections professionnelles est défini aux articles L. 2122-1 à 10 qui ne peuvent s'appliquer, fautes d'élections spécifiques, aux organisations patronales ${ }^{(7)}$.

Cet article est issu d'une recherche collective ${ }^{(8)}$ ayant donné lieu à la rédaction d'un rapport ${ }^{(9)}$ dont l'objectif n'était pas de mesurer la représentativité des organisations professionnelles d'employeurs mais de comprendre, en s'appuyant sur une analyse des normes et de leur processus d'élaboration, le rôle joué mais aussi les stratégies élaborées par l'Administration, le juge et les organisations patronales dans le processus de construction de la demande de représentativité. Plusieurs champs disciplinaires ont été investis : le droit, discipline à laquelle se rattache l'auteur de l'article, mais également 1'histoire du droit (afin de comprendre comment s'est formé le concept de représentativité) ou encore la sociologie, par l'étude de trois secteurs d'activité(10). Dix-neuf entretiens ont été

(7) L'audience est mesurée lors des élections professionnelles au sein de l'entreprise (comités d'entreprise, délégations uniques du personnel ou délégués du personnel).

(8) Réalisée par des chercheurs du laboratoire Droit et changement social (UMR CNRS 62977 / MSH Ange Guépin, Nantes). Ont participé à la rédaction du rapport de recherche Pascal Caillaud (juriste, chargé de recherche au CNRS), Stéphane Carré et Franck Héas (Maîtres de conférences en droit privé). Ont collaboré à la recherche Marion Blatgé (docteur en sociologie, Laboratoire Georges Friedmann [IDHE, UMR 8533/ISST-Université de Paris I, Panthéon-Sorbonne]), JeanPierre Chauchard (Juriste, professeur à la Faculté de droit de Nantes) et Jean Saglio (sociologue, retraité du CNRS - PACTE UMR CNRS/IEP/UPMF/UJF 5194).

(9) N. Maggi-Germain et J.-P. Le Crom (dir.), La construction de la représentativité patronale, rapport pour le Ministère de l'emploi, du logement et de la cohésion sociale / Dares, avril 2011, 402 p.

(10) Les huissiers de justice, le transport routier et l'économie sociale. réalisés en 2010 et au début de l'année 2011 auprès de dirigeants d'organisations patronales et de fonctionnaires. La mobilisation, au cours des entretiens, des sources documentaires étudiées en amont a eu l'avantage d'orienter le discours des enquêtés sur des pratiques. Ce travail préparatoire a permis de dépasser le cadre de discussions lisses et calquées sur des positions institutionnelles.

Ont également été analysés les travaux parlementaires des principales lois relatives au syndicalisme et à la négociation collective ainsi que les décrets et circulaires d'application, ces dernières présentant souvent un intérêt tout particulier. Un dépouillement, ici aussi systématique, des enquêtes de représentativité diligentées par l'administration du Travail a été réalisé (l'origine de ces enquêtes remonte à la mise en œuvre de la loi du 24 juin 1936 sur l'extension des conventions collectives).

Du point de vue du droit positif proprement dit, le travail a consisté en un dépouillement et une analyse de la jurisprudence du Conseil d'État et de la Cour de cassation, afin de déterminer la méthode et les biais par lesquels les juges concluent à la représentativité ou à l'absence de représentativité d'une organisation professionnelle d'employeurs. La problématique était donc de déterminer comment est reçue la représentativité patronale par les juges administratif et judiciaire, ce qui induisait une série de questions : quels sont les principes directeurs? Quelle est la méthode utilisée? Quels sont les critères sollicités? Le contentieux sur le sujet n'est pas si abondant : une vingtaine de décisions du Conseil d'État entre les années 1930 et 2011 ont été examinées; le contentieux de la représentativité patronale est plus restreint encore devant la Cour de cassation et ne concerne qu'une douzaine d'arrêts depuis 1970.

La vocation de cet article n'est pas de couvrir l'ensemble de la recherche réalisée - des articles, qui en développent certains thèmes particuliers, ont d'ailleurs été publiés (HÉAs, 2011; MAGGIGERMAIN, 2011) - mais d'apporter un éclairage sur un point fondamental : les fonctions et usages de la représentativité patronale. Être reconnu représentatif par l'Administration et/ou le juge confère à l'organisation patronale une légitimité particulière. La représentativité est d'abord une reconnaissance juridique qui confère certaines prérogatives (I) comme, par exemple, signer des conventions et accords susceptibles d'être étendus. Du point de vue de l'Administration, elle permet d'opérer une sélection entre les organisations professionnelles d'employeurs. Le rôle de l'Administration et du juge est, de ce fait, fondamental : la Direction générale du travail (DGT) du ministère du Travail peut, sous le contrôle du Conseil d'État, déterminer quelles sont les organisations professionnelles habilitées, d'un point de vue juridique, à engager l'ensemble des employeurs compris dans le champ 
d'application de la convention ou de l'accord. L'enjeu de la reconnaissance de représentativité dépasse le seul cadre des relations conventionnelles du travail : elle détermine, en partie, la structuration de l'économie par secteurs d'activités.

Cependant, les fonctions (les missions imparties par le droit) et les usages (l'utilisation qui est faite par les organisations ou l'Administration) de la représentativité se sont progressivement élargis (II) : le droit impose parfois la représentativité comme une condition pour siéger dans des instances décisionnaires de la branche, dans des instances publiques consultatives ou encore pour participer au paritarisme interprofessionnel. Si la représentativité constitue d'abord une forme de reconnaissance visà-vis de la profession, elle l'est aussi à l'égard de l'Administration : de facto ou de jure, elle confère une autorité pour peser dans les décisions publiques. $\mathrm{Si}$ un projet ou une proposition de loi devait être déposé afin de mesurer la représentativité des organisations professionnelles d'employeurs, comme cela a été fait en 2008 pour les organisations de salariés, ce ne pourrait être qu'en tenant compte de ces fonctions et usages ${ }^{(11)}$.

\section{La représentativité : une reconnaissance juridique}

La représentativité confère certaines prérogatives : elle permet à une organisation professionnelle d'employeurs de signer des conventions et accords collectifs de travail susceptibles d'être étendus et de siéger dans certaines instances.

\section{Être représentatif pour signer}

La notion d'organisation syndicale représentative s'analyse comme la construction historique d'une catégorie juridique qui a d'abord été pensée dans le cadre de la procédure d'extension des conventions et accords collectifs de travail.

(11) Pour de plus amples développements et des propositions concernant la mise au jour de critères de représentativité, $c f$. MAGGi-Germain (2011).

\section{Une représentativité consubstantielle à la procédure d'extension : éclairage historique}

Lorsque le mécanisme juridique de l'extension est créé, en $1936^{(12)}$, il permet au ministre du Travail d'étendre le champ d'application d'une convention ou d'un accord collectif de travail à l'ensemble de la branche d'activité; ce faisant, il leur reconnaît un effet erga omnes et non plus seulement inter partes : le mécanisme de l'extension rend obligatoire, sous certaines conditions, les stipulations d'un accord à l'ensemble des entreprises d'une branche professionnelle alors même qu'elles n'en sont pas signataires ou qu'elles ne sont pas adhérentes à l'organisation syndicale signataire. Sous l'empire de la loi du 25 mars 1919, la convention ou l'accord liait les parties : les représentants d'un syndicat ou d'une association professionnelle agissaient en vertu d'une délibération spéciale de leurs membres ou de mandats individuels et écrits de tous leurs membres. La loi de 1936 cherche, en créant le mécanisme de l'extension, à faire de la convention collective la loi de profession et un instrument de paix sociale, en obligeant (réglementer les conditions particulières du travail) mais aussi en empêchant (réduire les différentes formes de concurrence déloyale). Contrairement à la législation actuelle (art. L. 2261-19 cod. trav.), le mécanisme de l'extension ne vise alors que la branche d'activité ou la profession et est circonscrit aux secteurs de l'industrie et du commerce. La loi prévoit alors qu'à la demande d'une organisation syndicale patronale ou ouvrière «intéressée », le ministre du Travail réunit une commission mixte ${ }^{(13)}$ composée des représen-

(12) Loi du 24 juin 1936 modifiant et complétant le chapitre IV bis du titre II du livre $1^{\text {er }}$ du Code du travail : «De la convention collective de travail». Elle succède à l'accord de Matignon du 7 juin 1936 signé, dans un contexte de diminution des salaires et d'instabilité des emplois, de grèves et d'occupations d'usines, en particulier dans la métallurgie, entre Léon Jouhaux, Secrétaire général de la CGT, et M. Duchemin, Président de la CGPF, en présence de Léon Blum, nouveau Président du Conseil. En lien avec la crise de 1929 qui sévit aux États-Unis, elle fait partie d'un ensemble de cinq lois (loi du 20 juin 1936 sur les congés payés; loi du 24 juin 1936 sur la semaine de 40 heures; loi du 9 août 1936 sur la prolongation de l'obligation scolaire et l'âge d'admission des enfants dans les établissements commerciaux et industriels [reporté de 13 à $14 \mathrm{ans}$ ]; loi du 31 décembre 1936 sur les procédures de conciliation et d'arbitrage dans les conflits du travail; loi du 26 août 1936 sur les assurances sociales).

(13) En 1936, le Code du travail ne connaît que la commission mixte qui comprend alors les seuls représentants des organisations patronales et ouvrières. Mais l'intervention du ministre du Travail (ou de son représentant) est prévue en cas de désaccord, afin d'aider à la résolution du différend après avis de la section ou des sections professionnelles intéressées du Conseil national économique (art. $31 v b$ cod. trav.). Le droit établit aujourd'hui une distinction (codifiée par la loi du 13 novembre 1982 relative à la négociation collective et au règlement des conflits collectifs du travail - art. L. 133-1 $1^{\text {er }}$ et $2^{\mathrm{e}}$ al.) entre la commission paritaire de branche - art. L. 2261-19 cod. trav. - (qui correspond, en 1936 et dans les lois qui suivent, à la commission mixte) et la commission mixte paritaire de branche qui fait intervenir un représentant de l'État - art. L. 2261-20 cod. trav. 
tants des organisations syndicales, patronales et ouvrières, les plus représentatives de la branche d'industrie ou de commerce pour la région considérée ou, dans le cas où il s'agit d'une convention nationale, pour l'ensemble du territoire.

D’un point de vue doctrinal, cette période voit la formation d'une nouvelle théorie de la convention collective qui s'analyse comme une réglementation du travail à base contractuelle (LAROQUE, 1934, p. 103). C'est ce que la doctrine nommera, sous l'influence, en particulier, de Paul Durand, la thèse du dualisme juridique(14). Le contrat collectif est un «contrat réglementaire», dont l'objet est de réglementer d'une façon générale et uniforme le contenu des contrats individuels de travail conclus ultérieurement. Pour le Professeur BONNARD (1937, pp. 58 et s.), spécialiste du droit public, le contrat collectif n'est pas un contrat mais un «acte législatif élaboré par des organes privés et par voie conventionnelle». Cet engagement des signataires donne, suivant les termes de la circulaire d'interprétation de la loi de 1936, «[...] une valeur spéciale à la convention intervenue» ${ }^{(15)}$.

Les lois qui suivent confortent le mécanisme de l'extension de la couverture conventionnelle. Si la loi du 23 décembre 1946 constitue une parenthèse marquée par la mainmise de l'État sur les relations socioprofessionnelles ${ }^{(16)}$, l'après-guerre et la loi du 11 février 1950 annoncent le retour à la liberté conventionnelle. Le législateur englobe désormais dans le droit de la négociation collective des catégories jusque-là non visées par la loi de 1936 : salariés agricoles, gens de maison, offices publics et ministériels, professions libérales. Il s'agit alors d'aligner les régimes juridiques de l'ensemble des travailleurs.

Associant partenaires sociaux et représentants de l'État, la procédure d'extension obéit à des règles précises, définies par le Code du travail, qui conditionnent sa validité. La convention ou l'accord collectifs doit avoir été négocié dans le cadre d'une commission paritaire. Si un accord collectif peut être conclu sans que toutes les organisations patronales représentatives dans son champ d'application aient

(14) La convention collective est un "acte complexe où prennent place à la fois des obligations et des règles de droit» (DURAND, 1939, p. 358).

(15) Circulaire du ministre du Travail du 17 août 1936 relative à l'application de la loi du 24 juin 1936 sur les conventions collectives de travail, $J O$ du 3 septembre, p. 9392.

(16) La loi institue alors une hiérarchie entre les conventions régionales ou locales qui ne peuvent intervenir qu'après signature de la convention collective nationale dans la branche d'industrie concernée. «Le droit des conventions collectives vient désormais d'en haut. En même temps, les pouvoirs du ministre du travail sont renforcés» (Intervention de $\mathrm{M}^{\mathrm{me}}$ Marcelle Devaud, groupe des Républicains sociaux, rapporteur de la commission du travail et de la sécurité sociale, $J O$ déb. Parlement. Conseil de la République, séance du 26 janvier 1950, p. 271). Tous les accords doivent être agréés pour pouvoir entrer en vigueur. L'arrêté d'agrément vaut arrêté d'extension. Relativement peu de conventions seront signées sous l'empire de cette loi de circonstances. été invitées à la négociation, il faut que la représentativité de l'organisation signataire soit incontestable «dans le champ d'application considéré» pour prétendre à la représentation de l'ensemble du secteur d'activité. Autrement dit, c'est la structuration de la représentation patronale qui détermine le champ d'application de l'accord. Or cette structuration évolue, ce que montre très bien la jurisprudence s'agissant de la branche en particulier, «à géométrie variable» dès lors que son périmètre est d'abord déterminé par les parties. En sélectionnant les organisations professionnelles d'employeurs représentatives, l'Administration, lorsqu'elle accorde l'extension, et le Conseil d'État, lorsqu'il est amené à contrôler la légalité de l'arrêté d'extension, construisent le périmètre de la branche. S'agissant du niveau national interprofessionnel, la reconnaissance de représentativité des seuls Medef (Mouvement des entreprises de France), CGPME (Confédération générale des petites et moyennes entreprises) et UPA (Union professionnelle artisanale), c'est-à-dire d'organisations issues de l'industrie et du commerce, a donné naissance à la notion de «hors champ», utilisée en doctrine mais jamais consacrée par un texte. Les accords signés par des organisations syndicales autres que celles mentionnées précédemment ne peuvent prétendre à être étendus au niveau national et interprofessionnel. Pour autant, parce qu'ils n'appartiennent ni à l'industrie, ni au commerce, ces secteurs ne sont représentés par aucune des organisations professionnelles d'employeurs reconnues représentatives au niveau national et interprofessionnel par l'Administration et sont donc maintenus «hors champ», c'est-à-dire hors du champ d'application des accords signés au niveau national et interprofessionnel. Ce «champ» d'application, défini par les parties elles-mêmes sous le contrôle de l'Administration et du juge, est fortement corrélé à la structuration de la représentation patronale telle qu'elle résulte de l'histoire des relations professionnelles. La procédure d'extension a été pensée, en 1936, pour l'industrie et le commerce, les professions libérales ou l'agriculture étant exclues de son champ d'application jusqu'en $1950^{(17)}$. Sur l'ensemble des cinq lois votées à la suite des accords de Matignon du 7 juin 1936, seule celle sur les congés payés annuels s'applique à l'agriculture. Le «caractère très particulier» de l'agriculture est alors mis

(17) La loi du 11 février 1950 étend l'application des dispositions du Code du travail relatives à la négociation collective au secteur agricole et aux professions libérales. 
en avant dans les débats parlementaires ${ }^{(18)}$. La loi du 11 février 1950 procède à un rapprochement de l'agriculture tout en prenant en compte les particularismes de ce secteur. La motion préjudicielle ${ }^{(19)}$ visant à étendre le champ d'application du texte de loi est repoussée par une forte majorité au nom de la diversité des modes d'exploitation agricole ${ }^{(20)}$ et de la spécificité du travail agricole ${ }^{(21)}$. Ce particularisme se manifeste par la création, par amendement déposé par le groupe socialiste, d'une section spécialisée au sein de la commission supérieure des conventions collectives (aujourd'hui Commission nationale de la négociation collective $[\mathrm{CNNC}])^{(22)}$. La Fédération nationale des syndicats d'exploitants agricoles (FNSEA) et l'Union nationale des professions libérales (UNAPL) siègent de nos jours à la CNNC sans pour autant que l'on puisse les considérer comme des organisations représentatives au niveau national et interprofessionnel, comme c'est le cas du Medef, de la CGPME et de l'UPA. La structuration historique de la représentation patronale autour de l'industrie a conditionné et conditionne encore l'esprit des lois et les pratiques administratives.

(18) «L'article 1er que vous avez voté a consacré le droit à un congé minimum de quinze jours, comportant au moins douze jours ouvrables. Ce congé ne pourra être pris à la même époque dans toutes les régions de France. Les viticulteurs ne pourront pas accorder le congé au moment des vendanges; il en sera de même au moment des moissons pour les régions céréalières ", Alexandre Duval, député de la Fédération républicaine, intervention lors des discussions instituant un congé annuel payé dans l'industrie, le commerce, les professions libérales, les services domestiques et l'agriculture, Assemblée Nationale (AN), séance du 11 juin 1936, p. 1385.

(19) Motion $\mathrm{n}^{\circ} 72$ présentée par Jean Primet (groupe communiste). «Mais vous trouvez normal, et vous avez raison, d'établir une convention nationale dans l'industrie, où existent pourtant des différences beaucoup plus sensibles entre l'ouvrier mécanicien qui travaille aux usines Renault et celui qui travaille dans un petit garage de province. Vous refusez, par contre, d'établir une convention collective pour l'agriculture où les différences entre salariés sont certainement moins grandes», Jean Primet, groupe communiste, intervention devant le Conseil de la République, $J O$ débat. Parlement., séance du 27 janvier 1950 , p. 302

(20) Marcelle Devaud, présidente de la Commission du travail et de la sécurité sociale, groupe des Républicains sociaux, intervention devant le Conseil de la République, $J O$ débat. Parlement., séance du 27 janvier 1950, p. 302.

(21) «Il n'est pas possible d'admettre qu'un ouvrier agricole soit traité comme un ouvrier de l'industrie au regard des conventions collectives, car vous savez comme moi, monsieur Primet, que, dans beaucoup de cas, l'ouvrier agricole est l'auxiliaire du patron, que le plus souvent, il mange à sa table [...].», Henri Paumelle, groupe radical, membre de la Commission de l'agriculture et de la Commission du travail, intervention devant le Conseil de la République, $J O$ débat. Parlement., séance du 27 janvier 1950, p. 302.

(22) «Mais chacun sait aussi que les problèmes de salaires et les conditions de travail dans l'agriculture sont sensiblement différents des problèmes qui se posent dans les autres professions. Leur étude préalable au sein d'une section spécialisée nous paraît indispensable», Gérard Vée, socialiste, $2^{\mathrm{e}}$ séance du 4 janvier $1950, \mathrm{AN}, J O$, p. 106

\section{Formation de la catégorie juridique}

Comment obliger les tiers mais aussi les membres du syndicat signataire qui ne sont pas individuellement partie à l'accord, c'est-à-dire qui n'y ont pas consenti? Plusieurs conceptions s'opposent dès le début $\mathrm{du} \mathrm{Xx}^{\mathrm{e}}$ siècle et c'est en explorant les fondements philosophiques de la force obligatoire de la convention collective que la doctrine construit son raisonnement. Le fondement de l'obligation n'est pas la volonté (conception civiliste) mais «la suprématie du droit collectif sur le droit individuel» (RouAst, 1909, p. 86). Les conventions conclues lient les individus qui composent le syndicat en vertu de la théorie de la représentation. La convention collective est la manifestation d'une volonté collective, sans intermédiaire, dont la force obligatoire peut être rattachée à la capacité reconnue par le droit à une partie de représenter, au nom de l'intérêt collectif, une pluralité d'individus.

Si la notion d'organisations syndicales «les plus représentatives» est consacrée par la loi du 24 juin 1936 comme une condition sine qua non pour engager l'ensemble des employeurs d'une branche donnée, elle ne sera définie qu'avec la loi du 11 février 1950. La lecture des débats parlementaires de ces différentes lois, tout comme leurs circulaires d'application, montre que si le législateur n'a pas établi de distinction, lors de l'élaboration de cette catégorie juridique, entre les organisations professionnelles patronales et les organisations syndicales de salariés, la notion de représentativité a été pensée pour les organisations de salariés. Mais ce constat doit être relativisé : ce sont les mêmes critères qui seront utilisés, en tout ou partie, par l'Administration dans sa recherche de détermination de la représentativité des organisations patronales.

Consacrée par la loi de 1936, l'expression « organisations syndicales les plus représentatives », est reprise par les lois de 1950 et $1971^{(23)}$ mais non par celle de 1982, qui lui substitue l'expression « organisations syndicales d'employeurs et de salariés représentatives dans le champ d'application considéré» (24). L'expression «organisations syndicales les plus représentatives» fera débat lors des discussions préalables au vote de la loi de 1936, suscitant quelques difficultés d'interprétation. Au Sénat, un amendement de M. René Coty (repoussé par 189 voix contre 108) proposa de supprimer les mots «les

(23) Art. 12, II de la loi nº 71-561 du 13 juillet 1971 modifiant certaines dispositions du chapitre IV bis du titre II du livre Ier du Code du travail relatives aux conventions collectives de travail ainsi que certaines dispositions du titre II de la loi $\mathrm{n}^{\circ}$ 50-205 du 11 février 1950 modifiée, relative à la procédure de médiation, $J O$ du 14 juillet, pp. 6939-6941.

(24) Section I, art. 7 (art. L. 133-1 du Code du travail) de la loi $\mathrm{n}^{\circ} 82-957 \mathrm{du} 13$ novembre 1982 relative à la négociation collective et au règlement des conflits collectifs du travail, $J O$ du 14 novembre, pp. 3414-3421. 
plus »(25). La crainte est alors clairement évoquée d'instituer un monopole au profit de la CGT : «si vous voulez l'hégémonie d'une organisation de syndicats, alors repoussez mon amendement. Mais si vous voulez défendre la liberté syndicale conformément à la thèse qu'exposait $M$. Ramadier dans le passage que je me suis permis de placer sous vos yeux, je vous demande, Messieurs, de réserver à mon amendement un accueil favorable ${ }^{(26)} \gg$. C'est au nom de la liberté syndicale que certains prônent l'ouverture vers d'autres organisations :

«Cet article 31 va dispose, en effet, que la commission mixte dont vous savez le rôle capital, celle qui va élaborer les conventions collectives, ces sortes de codes à la Roosevelt qui vont non seulement régir les parties contractantes mais encore prendre force de loi pour l'ensemble d'une branche de commerce ou d'industrie, ces commissions mixtes, dis-je, qui sont ainsi à la base du projet, d'après les termes du deuxième alinéa de l'article 31 va, seraient composées des représentants des organisations syndicales, patronales et ouvrières les plus représentatives. [...]. Êtes-vous disposé, Monsieur le ministre du Travail, à donner à ce texte l'interprétation la plus libérale et à ne pas fermer la porte des commissions mixtes aux syndicats qui, sans être les "plus représentatifs", sont tout de même réellement, sérieusement représentatifs de tout ou partie, soit du patronat soit du personnel ? Quand vous aurez des syndicats, je le répète, qui ne seront pas des syndicats fictifs, ni des syndicats fantômes, mais des syndicats sérieux, êtes-vous disposé à leur donner accès à la commission mixte?»(27).

À l'inverse, pour le ministre du Travail JeanBaptiste Lebas, le pluralisme est source de «surenchère» (28) : «Ce que nous devons rechercher, que dis-je, exiger, c'est que les deux grands éléments qui se trouvent parfois aux prises, qui ont des différends, qui ont, hélas! assez souvent des conflits, soient bien représentés, qu'il y ait pour l'un ou l'autre élément des représentants indiscutables»(29). Ce sont alors deux conceptions de l'organisation socioprofessionnelle qui s'opposent, comme en témoigne cette intervention de Léon Blum, président du Conseil :

«Nous ne nous plaçons pas sur le même plan que les régimes totalitaires; nous n'essayons pas de faire une organisation de la profession par la volonté, sous l'ordre et sous le contrôle du pouvoir central. Cependant, nous tentons un commencement d'organisation et cette tentative est condamnée d'avance si, à l'intérieur des débats d'où sortiront les conventions collectives, si nous introduisons la multiplicité d'organisations patronales et d'organisations ouvrières déjà animées

(25) Sénat, séance du 17 juin 1936, JO du 18 juin, p. 522.

(26) Intervention de René Coty, sénateur membre du groupe de l'Union républicaine, Sénat, séance du 17 juin 1936, p. 523. (27) Idem, p. 522.

(28) Intervention de J.-B. Lebas, ministre du Travail, Sénat, séance du 17 juin 1936, p. 522

(29) Idem. les unes contre les autres d'un esprit de concurrence et de rivalité $\gg(30)$.

Les lois qui suivent, tout comme les pratiques administratives, s'inscrivent dans cette perspective de conciliation entre pluralisme syndical et exorbitance du droit de l'extension (31). Les textes adoptés témoignent d'une volonté «d'institutionnaliser» les organisations syndicales, et plus spécifiquement de salariés, dans un contexte de pluralisme d'après-guerre qui rompt avec $1936^{(32)}$. Cette institutionnalisation des organisations syndicales de salariés se traduit aussi par la volonté des parlementaires de briser d'éventuelles attaches politiques et par l'inscription, suite à un amendement déposé par le groupe communiste, dans le corps même de la loi de $1950^{(33)}$, des critères de représentativité. "La définition des principes du droit social ne peut être abandonnée à la volonté de groupements particuliers dont on ne peut que présumer le caractère représentatif. On ne peut laisser le soin de délibérer de mesures quasi-législatives à des parlements miniatures, qui seraient souvent des parlements croupions, surtout dans un pays où le droit social a toujours été plus légal que coutumier» ${ }^{(34)}$. L'exorbitance juridique de l'extension est contrebalancée par la condition de représentativité des organisations syndicales signataires. Si la négociation en commission paritaire composée de représentants des organisations syndicales d'employeurs et de salariés représentatives dans le champ d'application considéré constitue une condition essentielle (art. L. 2261-19 cod. trav.), de jurisprudence constante, un accord collectif peut être conclu sans que toutes les organisations patronales représentatives dans son champ d'application aient

(30) Intervention de L. Blum, président du Conseil, Sénat, séance du 17 juin 1936, JO du 28 juin, débat. Parlement. pp. 522 et $\mathrm{s}$.

(31) Exorbitance par rapport au droit civil qui limite les effets du contrat aux seuls signataires (art. 1165: «les conventions n'ont d'effet qu'entre les parties contractantes; elles ne nuisent point au tiers, et elles ne lui profitent que dans le cas prévu par l'article $1121 »-$ stipulation pour autrui).

(32) «En 1936, il y avait deux centrales syndicales importantes: la CGT et les travailleurs chrétiens. Aujourd'hui, la CGT s'est scindée en deux, et nous avons le CGT-Force ouvrière. En outre, sous le nom de CFTC, les syndicats chrétiens ont pris une importance qu'ils n'avaient pas en 1936. Nous avons, d'autre part, une centrale des indépendants et nous avons vu grandir en nombre et en autorité légitime la confédération générale des cadres», Marc Rucart, Rassemblement des gauches républicaines, intervention devant le Conseil de la République, JO débat. Parlement., séance du 26 janvier 1950, p. 278.

(33) Le projet de loi se réfère à la «réglementation en vigueur». C'est un amendement $n^{\circ} 48$ (adopté), déposé à la Chambre des députés en 1949 par le groupe communiste lors de la $1^{\text {e }}$ séance du 31 décembre qui lui substitue une liste de critères formant un nouvel alinéa de l'article $31 f$. Il prévoit que «la représentativité des organisations syndicales est déterminée d'après les critères suivants : les effectifs; l'indépendance; les cotisations; 1'expérience et l'ancienneté du syndicat; l'attitude patriotique pendant l'Occupation $»$.

(34) Intervention du rapporteur membre du groupe des Républicains sociaux, Marcelle Devaud, séance du 26 janvier 1950, JO, Conseil de la République, p. 269. 
été invitées à la négociation ${ }^{(35)}$. Mais encore faut-il que la représentativité de l'organisation signataire soit incontestable pour prétendre à la représentation de l'ensemble du secteur d'activité. Ce faisant, la reconnaissance de représentativité conférée via la procédure d'extension contribue indiscutablement à la structuration des rapports collectifs de travail.

Si la représentativité en tant que catégorie juridique a été pensée dans le cadre de l'extension, ses fonctions se sont progressivement élargies.

\section{Être représentatif pour siéger}

La représentativité est généralement la condition posée par le droit pour participer au paritarisme ou siéger dans des instances décisionnaires de la branche ou dans des instances publiques consultatives, l'exemple pouvant être donné par le secteur de l'agriculture.

\section{La participation au paritarisme}

Comme le montre le tableau ci-dessous, les fonctions attachées à la représentativité sont multiples et dépassent le champ de la négociation collective. Elle est une condition posée pour participer au paritarisme.

\section{Tableau : Exemples de fonctions attachées à la représentativité (début)}

\begin{tabular}{|c|c|}
\hline Champs d'intervention & Fonctions \\
\hline Négociation collective & $\begin{array}{l}\text { - Membre de la Commission nationale de la négociation collective (art. L. 2272-1 cod. trav.); } \\
\text { - Consultation obligatoire par le gouvernement, avant tout projet de réforme intéressant le travail, } \\
\text { l'emploi et la formation professionnelle (art. L. } 1 \text { cod. trav.); } \\
\text { - Demande d'extension d'une convention ou d'un accord, au sein d'une commission paritaire spécifique } \\
\text { (art. L. 2261-24 et L. 2261-19 cod. trav.). } \\
\text { Et pour les organisations de travailleurs spécifiquement : } \\
\text { - Négociation d'une convention ou d'un accord collectif de travail (art. L. 2231-1 cod. trav.); } \\
\text { - Désignation d'un délégué syndical dans l'entreprise ou l'établissement (art. L. 2143-3 cod. trav.); } \\
\text { - Déclenchement d'une grève dans les services publics (art. L. 2512-2 cod. trav.); } \\
\text { - Invitation à la négociation avant la conclusion ou la révision d'une convention ou d'un accord collectif } \\
\text { de travail (Cass. soc. } 8 \text { juillet 2009, JCP S 2009, 1468). }\end{array}$ \\
\hline $\begin{array}{l}\text { Service public de } \\
\text { l'emploi }\end{array}$ & $\begin{array}{l}\text { - Membre de Pôle emploi (art. R. 5312-7 cod. trav.); } \\
\text { - Membre des instances paritaires régionales de Pôle Emploi (art. R. 5312-28 cod. trav.); } \\
\text { - Membre de l'Unedic (art. L. 5427-1 cod. trav.); } \\
\text { - Détermination du régime d'assurance chômage (art. L. 5422-20 cod. trav.); } \\
\text { - Consultation à propos de la liste départementale des conseillers du salarié (en cas de licenciement), } \\
\text { établie par le Préfet (art. R. 1232-4 cod. trav.). }\end{array}$ \\
\hline $\begin{array}{l}\text { Formation } \\
\text { professionnelle }\end{array}$ & $\begin{array}{l}\text { - Création et gestion des Organismes paritaires collecteurs agréés (art. L. 6332-7 cod. trav.); } \\
\text { - Gestion du Fonds paritaire de sécurisation des parcours professionnels (art. L. 6332-18 cod. trav.); } \\
\text { - Membre de la Commission nationale de la certification professionnelle (art R. 335-24 Code de } \\
\text { l'éducation). }\end{array}$ \\
\hline Protection sociale & $\begin{array}{l}\text { - Participation aux organismes nationaux de sécurité sociale : CNAM (art. L. 221-3 cod. de la sécu. } \\
\text { soc.), CNAV (art. L. 222-5 cod. de la sécu. soc.), CNAF (art. L. 223-3 cod. de la sécu. soc.), ACOSS (art. } \\
\text { L. 225-3 cod. de la sécu. soc.); } \\
\text { - Participation aux organismes locaux de sécurité sociale : CPAM (art. L. 211-2 cod. de la sécu. soc.), } \\
\text { CAF (art. L. 212-2 cod. de la sécu. soc.), Urssaf (art. L. 213-2 cod. de la sécu. soc.), CRAM (art. } \\
\text { L. 215-2 cod. de la sécu. soc.); } \\
\text { - Membre du Conseil de la Caisse nationale de solidarité pour l'autonomie (art R. 14-10-2 Code de } \\
\text { l'action sociale et des familles); } \\
\text { - Participation (nomination d'un assesseur) aux Tribunaux du contentieux de l'incapacité (art. L. 143-2 } \\
\text { cod. de la sécu. soc.); } \\
\text { - Participation (nomination d'un assesseur) à la Cour nationale de l'incapacité et de la tarification de } \\
\text { l'assurance des accidents du travail (art L. 143-5 cod. de la sécu. soc.). }\end{array}$ \\
\hline
\end{tabular}

(35) Cass. Soc. 4 déc. 2007, n 06-43749, inédit, note Nadal S. (2008), «Organisations patronales représentatives : de quoi parle-t-on?», Revue droit du travail n ${ }^{\circ} 3$, pp. 189-190. 
Tableau : Exemples de fonctions attachées à la représentativité (fin)

\begin{tabular}{|c|c|}
\hline Champs d'intervention & Fonctions \\
\hline $\begin{array}{l}\text { Participation à des } \\
\text { instances en matière de } \\
\text { travail et/ou d'emploi }\end{array}$ & $\begin{array}{l}\text { - Commission nationale des inventions des salariés (art. R. 615-7 Code de la propriété intellectuelle); } \\
\text { - Conseil national de la formation professionnelle tout au long de la vie (art. D. 6123-2 cod. trav.); } \\
\text { - Conseil supérieur de la prud'homie (art. L. 1431-1 cod. trav.); } \\
\text { - Conseil d'orientation sur les conditions de travail (art. R. 4641-5 cod. trav.); } \\
\text { - Agence nationale pour l'amélioration des conditions de travail (art. L. 4642-2 cod. trav.); } \\
\text { - Haut conseil du dialogue social (art. R. 2122-1 cod. trav.); } \\
\text { - Conseil de surveillance du Fonds de réserve pour les retraites (art. R. 135-19 cod. de la sécu. soc.). }\end{array}$ \\
\hline $\begin{array}{l}\text { Participation à des } \\
\text { instances autres }\end{array}$ & $\begin{array}{l}\text { - Conseil national du bruit (art. D. 571-100 Code de l'environnement); } \\
\text { - Commission des comptes et de l'économie de l'environnement (art. D. 133-39 Code de } \\
\text { l'environnement); } \\
\text { - Conseils économiques et sociaux régionaux (CE } 26 \text { mai } 1982 \text { Union départementale des syndicats } \\
\text { chrétiens du Puy-de-Dôme, Recueil Lebon); } \\
\text { - Conseil national des politiques de lutte contre la pauvreté et l'exclusion sociale (art. R. 143-2 Code de } \\
\text { l'action sociale et des familles); } \\
\text { - Commission départementale des taxis et voitures de petite remise (art. } 3 \text { décret n } 86-427 \text { du } 13 \text { mars } \\
\text { 1986); } \\
\text { - Commission des droits et de l'autonomie des personnes handicapées (art. R. 241-24 Code de l'action } \\
\text { sociale et des familles). }\end{array}$ \\
\hline Financement & $\begin{array}{l}\text { - Possibilité de subventions de la part des communes ou de leurs groupements (art L. 2251-3-1 Code } \\
\text { général des collectivités territoriales). }\end{array}$ \\
\hline
\end{tabular}

Technique de gestion des relations socioprofessionnelles, le paritarisme est né dans les branches d'activité. D'inspiration patronale (Pollet, RENARD, 1995, p. 554), l'idée paritaire est d'abord liée à la question du travail : «Le souci de ne pas laisser "la fixation des salaires livrée aux hasards des discussions entre ouvriers et patrons ou à l'arbitraire de ces derniers", de "rendre plus faciles et plus fécondes les relations entre employeurs et employés, afin d'épargner au pays les conflits ouvriers" (Picard, 1921, p. 28 (36)) conduit à imposer une forme d'association patrons-ouvriers analogues à celle préconisée en Grande-Bretagne par la commission Whitley» (37). Des comités de salaires seront institués dans les années 1920. Ces fonctions seront ensuite reportées sur le mécanisme de l'extension (1936). Ce n'est que par la suite que l'idée paritaire pénètre le champ de la protection sociale (Pollet, ReNARD, 1995, p. 566). L'institution de la sécurité sociale donne naissance à une nouvelle forme de paritarisme qui «médiatise l'intervention de l'État» (Pollet, Renard, 1995, p. 546), faisant alors du paritarisme autant un mode de gestion des relations socioprofessionnelles qu'un projet politique. Il existe ainsi, pour reprendre la distinction établie par Robert LAFORE, un paritarisme «pur» et un paritarisme «de participation», octroyé par la puissance publique.

(36) Picard R. (1921), «Les progrès de la législation ouvrière en France pendant et depuis la guerre», Revue internationale du travail, $\mathrm{n}^{\circ} 1-2$, juillet-août, pp. 27-39.

(37) M. Whitley est un parlementaire libéral qui présida une commission chargée de réfléchir sur les conflits industriels. Elle rendit un rapport en 1917 qui permit la création, au sein des branches de l'industrie, de conseils composés de représentants des employeurs et des salariés chargés de discuter des salaires et des conditions de travail (Lemonnier B. [1998], «Les relations industrielles au Royaume-Uni de 1873 à 1970», Conférence donnée au Grand Amphithéâtre de la Sorbonne).
«D’un côté, un paritarisme "pur” peut être identifié : il repose sur la négociation entre partenaires sociaux, et se fonde en conséquence sur la convention collective nationale interprofessionnelle; logiquement, employeurs et salariés se partagent la gestion et leurs pouvoirs y sont très étendus, notamment en matière de fixation des contributions financières qui alimentent les organismes et sur les prestations que ces derniers versent; le contrôle de l'État n'est pas absent, mais dans l'épure théorique, il s'exerce en amont, via le mécanisme de l'agrément de la convention collective constitutive qui est requis pour lui faire produire tous ses effets de droit; les organismes de retraite complémentaire ainsi que le régime d'assurance chômage entrent indubitablement dans cette première catégorie. Il en est autre chose du paritarisme "de participation", paritarisme en réalité octroyé par la puissance publique et qui consiste à introduire, dans des institutions qui fonctionnent à l'initiative de l'État, des représentants des employeurs et des salariés à qui l'on confie des compétences gestionnaires plus ou moins étendues, mais toujours placées sous tutelle publique; cette seconde forme se retrouve très exactement dans les organismes de Sécurité sociale où les partenaires sociaux siègent seuls dans les conseils à l'exclusion de l'État; mais on peut identifier une autre sous-catégorie, avec l'ANPE et l'AFPA, structures dont les conseils sont tripartites, l'État y siégeant à côté des partenaires Sociaux» (LAFORE, 2001, p. 349).

La représentativité permet alors de conforter la légitimité des partenaires sociaux pour mieux asseoir le paritarisme.

Participer au fonctionnement d'instances paritaires créées par accord collectif suppose d'en être signataire ou d'y avoir adhéré. Le Conseil d'État 
refuse d'y voir une atteinte à la liberté syndicale ${ }^{(38)}$. La condition de représentativité peut ainsi être indirectement posée comme une condition pour siéger dans les instances de la profession mises en place par conventions ou accord étendus. L'adhésion d'une organisation d'employeurs représentative dans le champ d'application de la convention à une convention ou à un accord collectif emporte le droit de siéger dans les organismes paritaires et celui de participer à la gestion des institutions créées par la convention de branche ou l'accord professionnel ou interprofessionnel (art. L. 2261-4).

Les enjeux peuvent être conséquents, notamment en termes d'orientation de la profession, comme le montre, chez les huissiers, la participation à la gestion de l'École nationale de procédure (ENP), association créée par les partenaires sociaux (côté patronal, par la Chambre nationale des huissiers de justice, institution ordinale) par avenant du 7 octobre 1960 à la CCN (Convention collective nationale) du 15 mai 1959. Cette école est le lieu où se décide et où est mise en œuvre la formation continue, technique et pratique des salariés de la profession d'huissier de justice. La participation à la gestion de l'ENP constitue un enjeu majeur dans la maitrise des choix liés à l'organisation de la profession. Un chapitre Ier du Titre II de la convention collective nationale du 11 avril 1996, étendue par arrêté du 18 octobre $1996^{(39)}$, y est consacré. La reconnaissance, en 2007, de la représentativité de l'Union nationale des huissiers de justice (UNHJ) par l'Administration, après de multiples recours dont un devant l'Organisation internationale du travail, lui a permis d'y siéger. Un accord a été signé entre la Chambre et l'UNHJ afin de procéder à la répartition des sièges. Ce «protocole d'accord de partage des responsabilités » signé le 9 avril 2008 ${ }^{(40)}$, arrête, en ce qui concerne les «organismes paritaires», «le principe selon lequel les employeurs doivent être représentés pour moitié par des huissiers représentant la Chambre, pour moitié par des huissiers représentants la, ou les organisations syndicales d'employeurs représentatives de la profession»" (art. 3). Si les sièges du Conseil de direction sont répartis entre la Chambre et les représentants d'organisations syndicales représentatives (en l'espèce, l'UNHJ), la présidence demeure aux mains de la Chambre : "Il est expressément convenu et c'est une condition du présent protocole, que le président sera choisi par la Chambre» (art. 3.1).

La représentativité joue le rôle d'un sésame pour la gestion des institutions paritaires de branche ou interbranches, qu'il s'agisse des organismes de prévoyance prévus par le Code de la sécurité sociale (art. L. 931-1 et s. et R. 931-1-1 et s. cod.

(38) CE 31 mai 2002, no 229 574, Chambre nationale des professions libérales, publié au Recueil Lebon.

(39) $J O$ du 29 octobre 1996, p. 15824

(40) $N J H J \mathrm{n}^{\circ} 109$, mai-juin 2008 , p. 35, annexé, p. 357 sécu. soc.) ou des organismes paritaires collecteurs agréés (OPCA) chargés de la collecte des fonds de la formation professionnelle continue versés par les entreprises. La CARCO, Caisse de retraite complémentaire des clercs et employés des huissiers de justice, a été instituée par convention collective de branche du 11 avril 1996, étendue par arrêté du 18 octobre 1996. Il s'agit d'une institution professionnelle créée afin de gérer le régime de prévoyance obligatoire mis en place le $1^{\text {er }}$ juillet 1989 (accord du 29 juin 1989). Cette institution administre le second niveau du régime de retraite selon le système de la capitalisation collective et sert un complément aux prestations d'assurance vieillesse. Elle peut également proposer et gérer des produits à caractère facultatif, individuel ou collectif, au bénéfice des salariés ou retraités des offices, groupements et organismes professionnels des huissiers de justice (art. 3-4-1 de la CCN de 1996). L'avenant du 13 mai 2009 la transforme en caisse de retraite supplémentaire ${ }^{(41)}$. Les «membres adhérents» de la CARCO sont «les offices, groupements et organismes professionnels d'huissiers de justice entrant dans le champ d'application de la convention collective nationale du personnel des huissiers de justice et ayant à ce titre adhéré à l'institution» (art. 5 de l'avenant à la CCN du 14 mars $2008{ }^{(42)}$ ). Les «membres participants» sont les salariés ou anciens salariés. Le conseil d'administration de la CARCO est paritaire (représentants des membres adhérents et participants).

Dans le domaine de la gestion des fonds de la formation professionnelle, la création des OPCA est également une prérogative des organisations syndicales représentatives. L'organisme collecteur paritaire habilité à recevoir les contributions des employeurs est institué par accord conclu entre les organisations syndicales de salariés et d'employeurs représentatives dans le champ d'application de l'accord. Il a une compétence nationale, interrégionale ou régionale. Si son champ d'application est interprofessionnel, l'accord constitutif est valide et peut être agréé même s'il est signé, en ce qui concerne la représentation des employeurs, par une seule organisation syndicale (art. L. 6332-1 cod. trav.). Ce paritarisme donne lieu à un financement

(41) Avenant $n^{\circ} 36$ du 13 mai 2009 signé entre la CNHJ, l'UNHJ, d'une part, et la Fédération CFTC des commerces, du service et de la force de vente (CSFV-CFTC), la Fédération nationale des personnels des sociétés d'études et prévention CGT, la Fédération nationale de l'encadrement, du commerce et des services (FNECS-CGC) et la Fédération des services CFDT, d'autre part.

(42) Avenant $n^{\circ} 29$ du 14 mars 2008 portant modification d'articles de la convention collective signée entre la Chambre nationale des huissiers de justice, l'UNHJ d'une part, et d'autre part, le syndicat national des employés et cadres des professions judiciaires CSFV-CFTC, la Fédération nationale des personnels des sociétés d'études et prévention CGT, la Fédération nationale de l'encadrement du commerce et des services (FNECS) CGC, la Fédération employés et cadres FO, la Fédération des services CFDT. 
des organisations signataires de l'accord constitutif $(0,75 \%$ maximum des sommes collectées - art. $\mathrm{R}$. 6332-43 cod. trav.) (43) ainsi que des organisations interprofessionnelles d'employeurs représentatives au niveau national $(0,75 \%$ du montant des sommes collectées par les OPCA est reversé au FONGEFOR, association de gestion du fonds national de gestion paritaire de la formation professionnelle continue ${ }^{(44)}$, qui les répartit «à parité entre les OS de salariés et les organisations interprofessionnelles d'employeurs représentatives au niveau national»art. R. 6332-99). Sont exclues de ce financement, les organisations d'employeurs du «hors champ » (45).

Le paritarisme de participation s'exerce dans le cadre d'institutions multiples et variées : Haut conseil du dialogue social (art. R. 2122-1 cod. trav.), Fonds paritaire de sécurisation des parcours professionnels (art. L. 6332-18 cod. trav.), Commission nationale de la certification professionnelle (art R. 335-24 Code de l'éducation), Conseil national des politiques de lutte contre la pauvreté et l'exclusion sociale (art. R. 143-2 Code de l'action sociale et des familles), Pôle emploi (art. R. 5312-7 cod. trav.) et instances paritaires régionales de Pôle emploi (art. R. 5312-28 cod. trav.).

La représentativité permet aussi aux organisations professionnelles d'employeurs de se rapprocher des lieux d'exercice du pouvoir étatique.

\section{La participation aux instances décisionnaires ou consultatives : l'exemple du secteur de l'agriculture}

L'organisation économique de l'agriculture se présente comme une organisation intégrée, fortement marquée par une cogestion, «au centre de la politique de modernisation rapide de l'agriculture française de l'après-guerre» (Colson, 2008, p. 107). Elle est divisée en secteurs (viande, lait, vin, etc.) qui recouvrent l'ensemble des acteurs et des opérations (production, transformation, financement, distribution, etc.). Elle est en outre structurée, depuis les années soixante, autour des organisations de producteurs et des interprofessions, lieux de concertation entre les professionnels de chaque secteur (aquaculture et coquillages; fruits, légumes, pommes de terre et fleurs; grandes cultures et semences; lait et fromages; viandes, volailles et produits carnés; vins, spiritueux et autres boissons). Ces interprofessions constituent le cadre d'exercice

(43) Le «préciput» est utilisé pour les frais de missions et services qui sont effectivement accomplis par l'OPCA.

(44) Art. R. 6332-96 à 101 du Code du travail.

(45) Parce que non représentatives au niveau national et interprofessionnel (l'économie sociale, par exemple). de la représentativité (46) et les accords conclus dans ce cadre peuvent être étendus «dès lors qu'ils prévoient des actions communes ou visant un intérêt commun conformes à l'intérêt général et compatibles avec la législation de l'Union européenne» (art. L. 632-3 du Code rural). Leur extension par le ministre de l'Agriculture est subordonnée à l'adoption de leurs dispositions par une décision unanime des professions représentées dans l'organisation interprofessionnelle. À défaut, les accords ne concernant qu'une partie des professions représentées dans l'organisation interprofessionnelle sont adoptés à l'unanimité de ces seules professions, à condition qu'aucune autre profession ne s'y oppose (art. L. 632-4, 1er al. du Code rural). Constituées par les organisations professionnelles les plus représentatives de la production agricole, de la transformation, de la commercialisation et de la distribution (art. L. 632-1), ces «interprofessions » sont des personnes morales de droit privé, en général des associations régies par la loi du 1er juillet 1901. La loi du 10 juillet 1975 relative aux organisations interprofessionnelles agricoles (art. L. 632-1 à 11 du Code rural) les a habilitées à prélever, sur tous les membres des professions les constituant, des cotisations résultant des accords étendus (art. L. 632-6). Il résulte parfois de la forte intégration du secteur associée à la captation de la représentation dans ces interprofessions par des (une?) organisations professionnelles d'employeurs porteuses d'un certain projet économique, une consanguinité aux effets pervers, comme cela a pu être souligné en 2002 par la Cour des comptes (47) s'agissant de la filière des oléo-protéagineux ${ }^{(48)}$.

Parallèlement à ce système économique intégré, qui fait appel à des organisations représentatives au sein des organisations interprofessionnelles, il existe aussi une forme de participation aux instances publiques consultatives, nationales, régionales ou départementales issue d'une "pratique institutionnelle [qui] s'est imposée à la fin des années 1960 et concrétisée par les conférences annuelles à partir de

(46) D'où la difficulté pour certaines organisations patronales non-implantées dans les interprofessions mais représentatives au niveau national interprofessionnel à peser à ce niveau. Bien qu'elles représentent à elles deux près de $40 \%$ du collège électoral agricole, ni la Confédération paysanne, ni la Coordination rurale ne siègent dans les interprofessions. Elles ont obtenu respectivement $20,5 \%$ et $18,7 \%$ des voix aux dernières élections des chambres d'agriculture, en 2007 (cf. Van Eeckhout L. [2010], «La diversité du syndicalisme agricole contestée», Le Monde 5 oct. 2010, p. 14). Ces organisations syndicales, qui n'existaient pas lorsque le secteur de l'agriculture a été organisé en interprofessions, refusent aujourd'hui de verser les COV, cotisations volontaires obligatoires, sans avoir droit, en retour, à faire entendre leur voix dans l'interprofession.

(47) Cour des comptes, Rapport 2002, «L'utilisation de "cotisations volontaires obligatoires" prélevées sur les producteurs d'oléo-protéagineux », pp. 579-596, p. 580 en particulier.

(48) Essentiellement le soja, tournesol, colza, pois, fèves et lupins. 
1971 et les mardis mensuels, rendez vous devenus rituels entre le ministre de l'Agriculture et les présidents des quatre grandes organisations professionnelles agricoles (OPA) constituées en Conseil de l'agriculture française (CAF)» (Colson, 2008, p. 108) composé de représentants de la FNSEA et de sa section jeunes, le CNJA (Centre national des jeunes agriculteurs - aujourd'hui les Jeunes agriculteurs), de la Confédération nationale de la mutualité, de la coopération et du crédit agricoles (CNMCCA) et du président de l'Assemblée des Chambres d'agriculture.

Ce «modèle de partenariat» (Colson, 2008, p. 108) sollicite d'autres critères de représentativité. Ces instances administratives, placées auprès du ministre chargé de l'Agriculture, sont nombreuses : Conseil supérieur d'orientation et de coordination de l'économie agricole et alimentaire (CSO) ${ }^{(49)}$, Conseil national de l'alimentation, Conseil national des baux ruraux, Conseil supérieur des prestations sociales agricoles (CSPSA) ${ }^{(50)}$, etc. Cette participation du secteur de l'agriculture est institutionnalisée : elle a en effet été posée en principe par la loi d'orientation agricole, modifiée, du 9 juillet $1999^{(51)}$ qui prévoit, en son article 2, I, que «l'ensemble des organisations syndicales d'exploitants agricoles qui remplissent les conditions fixées par décret en Conseil d'État ont vocation à être représentées au sein des commissions ainsi que dans les comités professionnels ou organismes de toute nature investis d'une mission de service public, ou assurant la gestion de fonds publics ou assimilés, où siègent des représentants des exploitants agricoles». Le décret du 28 février $1990^{(52)}$, modifié par le décret

(49) Créé par la loi du 4 juillet 1980 d'orientation agricole, «il participe à la définition de la politique nationale d'orientation des productions» (art. 4 du Titre II de la loi). Y siègent, notamment, au titre des représentants professionnels de la production agricole : la Confédération nationale de la mutualité, de la coopération et du crédit, la FNSEA, la Confédération paysanne, le Président de l'assemblée permanente des chambres d'agriculture, les Jeunes agriculteurs, la Coordination rurale. (art. R. 611-1 à R. 611-3 et D. 611-4 à D. 611-17 cod. rural). Il se prononce à la majorité des membres présents ou représentés. (50) Il examine tous les sujets relatifs à la protection sociale agricole. La formation plénière comprend, notamment, un représentant de chacune des organisations syndicales à vocation générale d'exploitants agricoles habilitées en application de l'article 3 du décret $n^{\circ} 90-187$ du 28 février 1990 relatif à la représentation des organisations syndicales d'exploitants agricoles au sein de certains organismes ou commissions, désigné sur leur proposition (art. D. 721-3 cod. rural).

(51) Loi n ${ }^{\circ} 99-574, J O$ du 10 juillet, p. 10231.

(52) Décret $n^{\circ}$ 90-187 du 28 février 1990 modifié relatif à la représentation des organisations syndicales d'exploitants agricoles au sein de certains organismes ou commissions, $J O$ du $1^{\text {er }}$ mars 1990, p. 2585. du 16 février $2000^{(53)}$, définit les conditions de leur participation aux différentes commissions, comités professionnels ou organismes départementaux :

« $1^{\circ}$ Justifier d'un fonctionnement indépendant, régulier et effectif depuis cinq ans au moins;

$2^{\circ}$ Avoir obtenu dans le département plus de $15 \%$ des suffrages exprimés lors des élections à la chambre d'agriculture (collège des chefs d'exploitation et assimilés); lorsque deux organisations syndicales ont constitué une liste d'union ayant obtenu plus de $30 \%$ des suffrages, elles sont réputées satisfaire l'une et l'autre à cette condition.

La condition d'ancienneté prévue au $1^{\circ}$ ci-dessus est remplie par une organisation issue de la scission d'une organisation remplissant elle-même cette condition ou de la fusion d'organisations dont plus de la moitié remplissaient cette condition.»

(Art. $1^{\text {er }}$ du décret.)

Ces organisations représentatives au sens du décret de 1990 sont habilitées à siéger dans les multiples instances paritaires qui structurent le secteur de l'agriculture.

Il y existe ainsi trois formes de représentativité : l'une permettant de représenter «l'interprofession », une autre permettant de siéger dans certaines instances consultatives ou décisionnaires et enfin une dernière destinée à signer des conventions et accords collectifs susceptibles d'extension. Il n'existe pas, en l'état actuel du droit, de «passerelles», comme l'explique la circulaire du 21 octobre 2009 adressée aux préfets de région, aux préfets de département, aux directeurs régionaux du travail, de l'emploi et de la formation professionnelle : «Certaines organisations d'exploitants reconnues représentatives aux termes du décret de 1990, peuvent exciper de cette reconnaissance pour participer à la commission mixte paritaire. Face à ce type d'exigence, il convient d'être vigilant et, éventuellement, de consulter l'administration centrale» ${ }^{(54)}$.

Si les textes imposent la représentativité comme condition pour siéger dans certaines instances, les organisations professionnelles l'utilisent aussi dans des buts bien précis.

(53) Décret $\mathrm{n}^{\circ}$ 2000-139 du 16 février 2000 fixant les conditions de représentation des organisations syndicales d'exploitants agricoles au sein de certains comités, commissions ou organismes et modifiant le décret $\mathrm{n}^{\circ}$ 90-187 du 28 février 1990 relatif à la représentation des organisations syndicales d'exploitants agricoles au sein de certains organismes ou commissions.

(54) Circulaire du ministère de l'Alimentation, de l'agriculture et de la pêche et du ministère du Travail (SG/SAFSL/SDTPF/ C2009-1525, DGT/N2009/23) du 21 octobre 2009, précitée, p. 5 . 


\section{Les multiples usages de la représentativité}

Pourquoi des organisations professionnelles d'employeurs, dont l'existence est parfois ancienne, se décident-elles à présenter au ministre du Travail une demande de reconnaissance de représentativité? Pourquoi certaines organisations patronales contestent-elles, lors de la procédure d'extension, la représentativité d'autres organisations? Derrière la reconnaissance (ou l'absence de reconnaissance) juridique se nouent des jeux de pouvoirs et de positionnement. Se faire reconnaître représentatif par les services du ministère du Travail prend alors tout son sens : il permet d'être reconnu des pairs et des tiers. Pour l'Administration, la reconnaissance de représentativité lui offre le moyen d'intervenir, sous le contrôle du juge administratif, dans la structuration des secteurs d'activité.

\section{Se faire reconnaître représentatif pour être reconnu}

Se faire reconnaître représentatif permet aux organisations patronales de positionner un secteur d'activité, comme ce fut le cas pour l'économie sociale au niveau national et interprofessionnel, mais également de se positionner au sein d'un secteur d'activité, autrement dit d'affirmer une identité au sein de la branche. De ce point de vue, la reconnaissance de représentativité permet aussi de se démarquer d'autres organisations patronales.

\section{Le positionnement d'un secteur d'activité : l'exemple de l'économie sociale}

L'exemple de l'économie sociale (les mutuelles, les associations, les coopératives et les fondations) montre de manière assez éclairante le lien qui existe entre représentativité et structuration d'un secteur d'activité. Représentatives au sein de plusieurs branches d'activité, les organisations professionnelles d'employeurs de l'économie sociale souhaitent que leur représentativité soit reconnue au niveau national interprofessionnel. Face au refus des services du ministre du Travail, un recours a été déposé devant le Conseil d'État.

Le rassemblement de la représentation patronale a d'abord dû se réaliser au sein des secteurs qui la composent. Les trois grandes organisations d'employeurs de l'économie sociale sont le Groupement des entreprises mutuelles d'assurance (Gema), l'Union des fédérations et syndicats nationaux d'employeurs sans but lucratif du secteur sanitaire, médico-social et social (Unifed) et l'Union de syndicats et groupements d'employeurs représentatifs dans l'économie sociale (Usgeres). Si elles sont parvenues à se rassembler, l'unification semble un objectif difficile à atteindre mais essentiel afin de «peser plus dans les débats». L'extrait de cet entretien réalisé en 2008 (MagGi-Germain, 2009) avec un représentant d'une fédération d'employeurs du secteur sanitaire, social et médico-social à but non lucratif résume très bien l'esprit dans lequel sont les organisations professionnelles patronales de l'économie sociale :

«À l'origine, il y avait, d'une part, la branche sociale, et d'autre part, la branche sanitaire, elle relevait de la convention collective de 51. Il y avait deux branches distinctes et, depuis les années quatre-vingt-dix, il y a eu la volonté de faire se rejoindre les deux branches, de façon à former un pôle d'attractions plus fort parce que le secteur social, par lui-même, était quand même, jusqu'à il y a quelques années, était quelque chose d'assez marginal, quelque chose d'assez tenu à l'écart parce que, par exemple, le ministère du Travail ne nous a guère consultés sur les réformes législatives en matière de droit du travail.»

(Représentant d'une fédération d'employeurs du secteur sanitaire, social et médico-social à but non lucratif.)

Le parcours vers la reconnaissance de représentativité au niveau national interprofessionnel s'inscrit dans un processus de reconnaissance des spécificités du secteur. Les "voix de l'économie sociale» ne sont pas - ou pas complètement - portées par les organisations patronales reconnues représentatives au niveau national interprofessionnel, comme l'explique le même intervenant :

«Beaucoup de lois qui ont été votées, les lois Auroux,
les lois sur la durée du travail, c'est quand même des
lois qui étaient plutôt destinées aux secteurs industriel
et commercial et qui souvent étaient fort peu adaptées
à nos établissements et services, qui souvent sont des
établissements qui fonctionnent 24 heures sur 24, avec
du travail de nuit, des spécificités très particulières.
En plus, pour des raisons historiques, philosophiques
et autres, le secteur sanitaire et social n'a jamais
été représenté au sein du Medef, qui auparavant,
s'appelait CNPF et donc, de ce point de vue-là, les
besoins du secteur social et sanitaire ont quand même
été méconnus pendant longtemps. C'est pour ça qu'ily
a eu, durant les années quatre-vingt-dix, la volonté de
créer un secteur sanitaire et social qui soit beaucoup
plus représenté, beaucoup plus structuré.»

La rupture identitaire est parfois expressément affirmée et l'adhésion à une organisation d'employeurs autre que le Medef ou les organisations professionnelles «historiques» relève d'un acte d'engagement fort. Une illustration peut être donnée par l'adhésion, en 2009, du SyNOFDES, Syndicat national des organismes de formation de l'économie sociale, au Ceges, Conseil des entreprises et groupements de l'économie sociale dont la vocation est de fédérer la représentation patronale dans le secteur de l'économie sociale. Le syndicat intervient dans le champ de la formation professionnelle continue, là où existe déjà la Fédération de la formation professionnelle, adhérente au Medef. Sa 
création en $2007^{(55)}$ par la fédération des UROF ${ }^{(56)}$, des INFA ${ }^{(57)}$, des INFREP ${ }^{(58)}$ et des INSTEP(59) est motivée par un rejet du Medef, comme cela est clairement expliqué sur le site internet du SyNOFDES : «Pourquoi la création du SyNOFDES? Parce que ${ }^{(60)}$ les employeurs de l'économie sociale ne peuvent pas, statutairement et juridiquement, être représentés par le Medef et que la FFP (Fédération de la formation professionnelle) syndicat employeur de notre secteur, ne peut pas, du fait de son appartenance, représenter les employeurs de l'économie sociale.» ${ }^{(61)}$

Le secrétaire général de l'Usgeres confirme :

«Il y a aussi un enjeu par rapport à la convention collective de la branche de la formation professionnelle. La fédération de la formation professionnelle étant au Medef, et ces organisations-là se reconnaissant dans des organisations qui émargent sur la commande publique, donc plutôt dans l'économie sociale et solidaire, se reconnaissent davantage dans des modes d'entreprendre de l'économie sociale et solidaire et ont décidé de constituer leur propre champ d'application qui va se traduire dans la constitution de leur propre branche professionnelle.»

On peut parler d'effet repoussoir du Medef. Cela se vérifie tout particulièrement s'agissant du secteur sanitaire, social, médico-social à but non lucratif, comme le montre cet extrait d'entretien :

«Bon, vous n'ignorez pas, ou vous l'ignorez peut-être, qu'au Medef, il y a des activités de notre secteur, qui est le secteur hospitalier, qui sont à but lucratif. Et on a peut-être pas tout à fait la même approche des relations sociales.»

(Un président de l'Unifed.)

Cet effet repoussoir est accentué par les évolutions du secteur médico-social. En affirmant leur identité, les employeurs de la branche entendent marquer leurs distances avec le patronat du secteur lucratif, qui prend aujourd'hui en charge une partie significative des activités du secteur. C'est donc, la capacité du collège des employeurs à représenter l'ensemble des entreprises qui se trouve mise en cause.

Ces extraits d'entretiens font clairement apparaître à quel point la reconnaissance de représentativité permet de réaliser un équilibre entre

(55) AG constitutive du $1^{\text {er }}$ mars.

(56) Fédération nationale des unions régionales des organismes de formation; fondée en 2000, elle regroupe des organismes de formation intervenant pour l'essentiel sur la commande publique de formation (http://www.federation-urof.org/).

(57) Institut national de formation et d'application.

(58) L'Institut national de formation de la Ligue de l'enseignement (http://www.infrep.org/).

(59) Organisme de formation et d'aide aux entreprises dans le domaine de la formation.

(60) En gras dans le texte.

(61) http://www.synofdes.org/01_presentation.php les représentations ${ }^{(62)}$. Cet équilibre est d'autant plus important à atteindre que la représentativité conditionne, d'un point de vue juridique, la représentation au sein des organismes paritaires. Par-delà les valeurs portées par l'économie sociale, c'est un autre modèle économique qui est proposé. Mais l'affirmation identitaire ne se fait pas contre les organisations professionnelles patronales historiques, ce que l'un de nos interlocuteurs, un représentant de l'Usgeres, appelle le "patronat classique». L'économie sociale illustre cette volonté d'être reconnu représentatif pour être représenté. Elle se retrouve également dans d'autres secteurs, comme les professions libérales, ainsi que le montre cet extrait d'entretien relativement long, mais très clair, réalisé avec un dirigeant de l'UNAPL :

«Donc, on a une problématique : nous sommes en moyenne des TPE [Très petites entreprises], très proches de nos salariés mais nos salariés ne sont quand même pas les mêmes que ceux de l'artisanat. Chez nous, la plupart de nos employés - puisqu'il n'y a pas d'ouvriers - ont au moins le baccalauréat et quelquefois, le même titre que nous, et ils peuvent être salariés avec le même titre. Donc, on a des besoins qui sont différents. Dans l'ANI [Accord national interprofessionnel], on va discuter de choses pour ces trois-là et pour les cinq centrales, qui sont un peu différentes de ce qu'on va avoir comme besoins. Je me déplace dans des tas de réunions où on vous parle de droit social, de droit à la formation, et quand je vois qu'on fait venir comme représentants du terrain des gens de chez Veolia, chaque fois je me mets en rogne et je suis obligé de le dire, je dis : "Écoutez, moi, je veux bien qu'on me présente des pistes de réformes de la formation professionnelle, mais que ce soit fait par des gens de chez Veolia... chez nous, il y a pas de nettoyeur de tuyaux, en revanche, pour les salariés de nos entreprises, on est amputé et je suis pas d'accord!'. Par ailleurs, il faut quand même qu'on développe notre champ conventionnel, qu'on fasse valoir nos besoins, qu'on les traite, les besoins de nos salariés dans nos entreprises libérales... Il nous faut, à la fois, être présents dans les ANI parce qu'après, on nous l'impose au travers de la loi et puis, quand même, développer nos facettes propres.»

\section{Le positionnement dans un secteur d'activité}

Être reconnue représentative permet à une organisation professionnelle d'employeurs de signer des conventions et accords collectifs susceptibles d'être étendus. La représentativité permet alors de maîtriser le sort de la branche d'activité ${ }^{(63)}$. Un syndicat

(62) Sur l'articulation entre les concepts de représentation et de représentativité, se référer à notre rapport, précité, pp. 24-31. (63) Comme l'explique le commissaire du gouvernement Pierre Nicolaÿ dans ses conclusions : l'activité de la pose des vitres, glaces et vitrines « $[. .$.$] ne peut prétendre à la qualité de$ branche d'activité et qu'ainsi les organisations syndicales qui la représentent ne peuvent prétendre être maîtresse de son sort». Pierre Nicolaÿ, Commissaire du gouvernement, conclusions sous CE 2 mars 1962, Union des Chambres syndicales de miroitiers, Droit social, ${ }^{\circ} 12$, pp. 604-608, p. 607. 
patronal peut ainsi demander à être reconnu représentatif contre une autre organisation afin d'être en capacité de peser dans les négociations de branches. L'exemple peut être donné de l'Optique-Lunetterie où les grandes enseignes, qui ont quitté le syndicat patronal «historique», ont ensuite contesté sa représentativité. En l'état actuel du droit, ce sont les pratiques administratives qui, s'appuyant sur des textes conçus pour les organisations syndicales de salariés, déterminent la représentativité des organisations patronales. Une organisation minoritaire représentative au niveau d'une branche peut ainsi engager l'ensemble des employeurs par sa signature, sans droit d'opposition pour les non-signataires. On peut alors émettre l'hypothèse qu'une demande de reconnaissance de représentativité qui entraîne une scission au sein de la branche et conduit à la création d'une nouvelle branche résulte de l'impossibilité pour certaines organisations patronales à faire entendre leur voix (l'exemple est donné par les experts-chimistes dans les années soixante ${ }^{(64)}$ ou, de nos jours, par les installateurs de cuisines qui souhaiteraient être reconnus comme branche $\left.{ }^{(65)}\right)$. Mesurer la représentativité des organisations professionnelles d'employeurs et ouvrir un droit d'opposition aux accords et conventions collectifs signés par une ou plusieurs organisations minoritaires constitue alors un enjeu essentiel des réformes à venir (MAGGi-Germain, 2011).

La demande de reconnaissance de représentativité constitue un moyen pour peser dans un secteur d'activité et, ce faisant, se démarquer des organisations patronales en place. L'exemple peut être donné, dans le secteur des professions libérales, par la Coordination des associations de professionnels indépendants libéraux (CAPIL), association loi de 1901 non encore déclarée officiellement (les statuts et le règlement intérieur sont en cours de constitution) ${ }^{(66)}$ qui regroupe exclusivement des associations, unions ou syndicats professionnels de professionnels indépendants ${ }^{(67)}$. Elle n'a donc pas, pour l'instant, d'existence juridique. Elle a été créée

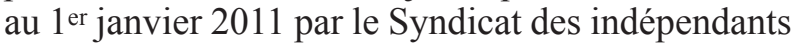
consultants et formateurs-Fédération des formateurs consultants (Sicfor-FCF), l'Association professionnelle des métiers de la traduction (Aprotrad) et par Freelance en Europe. Son objectif est la promotion du travail indépendant en France et en Europe et la défense des intérêts des professionnels qui l'exercent en se démarquant des professions libérales

(64) N'étant pas affiliés à l'Union des industries chimiques, principale organisation signataire de la convention, étaient-ils pour autant représentatifs au sein d'une nouvelle branche? $C f$. notre rapport, pp. 161 et s.

(65) L'UNICPRO (Union nationale des installateurs de cuisines professionnelles) a été créée en janvier 2011.

(66) Informations communiquées par le site internet de la CAPIL : http://www.coordination.pro/CapilWeb4.html (consulté le 26 février 2012).

(67) Suivant le protocole d'accord institutif signé par les organisations professionnelles fondatrices (art. 2.2). réglementées (médecins, dentistes, experts comptables, architectes, etc.) ou des autres professionnels tels les agriculteurs, commerçants ou artisans ${ }^{(68)}$. Il s'agit de prestataires intellectuels indépendants définissant eux-mêmes leurs conditions de travail avec leurs clients, et réalisant leur mission sans aucun lien de subordination, conformément aux dispositions de 1'article L. 8221-6, I, du Code du travail. «Ni agriculteurs, ni commerçants, ni artisans, ni professionnels libéraux réglementés, ils exercent toutes les professions hier purement salariées : ingénieurs, consultants, traducteurs, graphistes, formateurs, informaticiens, secrétaires, communicants, etc., sous toutes les formes sociales disponibles : autoentrepreneurs, entreprises individuelles, sociétés, auteurs (Agessa, Maison des Artistes) et même sous des formes "assimilées salariées" (journalisme, reportage photographique, portage salarial)» (69). Il n'existe aujourd'hui, pour la CAPIL, aucune structure officielle les représentant. «Les Professionnels Indépendants Libéraux ne possèdent aucun organe de représentation globale. En effet, les organisations représentant les professionnels libéraux, UNAPL (Union nationale des associations de professionnels libéraux) et CNPL (Chambre nationale des professionnels libéraux) représentent essentiellement les professions réglementées qui, elles, disposent par voie réglementaire d'Ordres ou de Chambres hors desquels elles ne peuvent être exercées» ${ }^{(70)}$. Un protocole d'accord a été adopté concomitamment à la création de la CAPIL et sera soumis à toute association, syndicat ou groupement divers souhaitant participer à cette Coordination. Son objectif principal est de représenter les «professionnels indépendants libéraux » auprès des instances réglementaires, nationales et régionales, afin de faciliter le travail indépendant (art. 1.1 de l'accord). «Les objectifs secondaires sont de représenter les professionnels indépendants libéraux auprès de toutes les instances intervenant dans le monde social et économique, national ou international» (art. 1.2 de l'accord). Elle constitue «une première étape dans l'organisation d'une structure permanente de représentation des professionnels indépendants» (art. 2.1). Ils se reconnaissent comme travailleurs indépendants car ils n'emploient aucun salarié(71). Leur vocation n'est pas de participer à des négociations collectives mais de peser sur la décision publique. Leur positionnement peut être qualifié de positionnement «à rebours» des autres organisations professionnelles. Ils ne se fédèrent pas autour des valeurs communes des professions libérales telles

(68) $C f$. «Le Sicfor-FCF s'organise avec les professionnels indépendants», Le quotidien de la formation (publication du Centre Inffo), $\mathrm{n}^{\circ} 1447,18 / 01 / 2011$.

(69) Source: http : //www.coordination.pro/

(70) Dossier de presse diffusé par la CAPIL, «Pourquoi créer une coordination?», p. 4, mis en ligne sur le site début janvier 2011 (http://www.coordination.pro/Dossier_presse.pdf).

(71) Dossier de presse diffusé sur le site web de la CAPIL, «Qui sont les professionnels indépendants libéraux?», p. 1. 
qu'elles sont notamment définies par le Ceplis ${ }^{(72)}$ (confidentialité, participation à la formation professionnelle continue, indépendance et impartialité, honnêteté et intégrité, etc. $\left.{ }^{(73)}\right)$. Même s'ils déclarent les respecter, ils «sont extrêmement réticents à les associer à la définition même de leurs professions » et rejettent l'idée même d'une organisation ordinale de la profession : «Il n'est nul besoin de tribunal, fut-il de pairs, pour exiger le respect de ces règles. [...] comment un traducteur, un graphiste ou un informaticien étranger, ou français résidant au-delà des frontières, pourrait-il être empêché d'exercer son métier par une quelconque organisation purement franco-française?» (74). La CAPIL demande à être reconnue par les pouvoirs publics, notamment au sein de la Coordination nationale de concertation des professions libérales (CNCPL) : «La Coordination appelle les pouvoirs publics à prendre en compte leur existence, en particulier au sein de la Coordination nationale de concertation des professions libérales (CNCPL) qui élabore en ce moment une définition positive des professions libérales» ${ }^{(75)}$. Instituée par le décret du 3 janvier 2003 (76), la Commission nationale de concertation des professions libérales a été remplacée par la Commission nationale des professions libérales par le décret du 21 février $2011{ }^{(77)}$. Elle est placée auprès du ministre chargé des professions libérales. Elle peut être consultée préalablement à tout projet de texte concernant les professions libérales (art. 2 ); elle examine, «dans le respect des prérogatives des instances ordinales et syndicales », toutes les questions intéressant les professions et les activités libérales (art. 3). Elle est composée de représentants de différents ministres (Justice, Santé, Culture, Équipement, Budget, Agriculture, Éducation nationale), de quatorze personnalités qualifiées et de représentants des professions libérales désignés par les syndicats des professions libérales, représentants «issus des professions réglementées et non réglementées, proposés par l'Union nationale des professions libérales, après consultation des organisations professionnelles concernées »

(72) Conseil européen des professions libérales, émanation de l'UNAPL (Union nationale des associations de professionnels libéraux) et de la CNPL (Chambre nationale des professionnels libéraux). Il s'agit d'une association interprofessionnelle représentant les professionnels libéraux au niveau communautaire, organisation internationale non gouvernementale et à but non lucratif enregistrée sous le droit belge. Le président du bureau exécutif est issu de l'UNAPL.

(73) Déclaration adoptée à l'unanimité par tous les membres du Ceplis lors de l'Assemblée générale tenue à Bruxelles le 20 juin 2007.

(74) Dossier de presse diffusé par la CAPIL, «Pourquoi créer une coordination?», précité, p. 4.

(75) http://www.coordination.pro/

(76) Décret $n^{\circ} 2003-15$ du 3 janvier 2003 portant création de la Commission nationale de concertation des professions libérales, $J O$ du 5 janvier, p. 340 .

(77) Décret $n^{\circ}$ 2011-200 du 21 février 2011 portant création de la Commission nationale des professions libérale, $J O \mathrm{du}$ 23 février, p. 3318. (dix-huit représentants), par la Chambre nationale des professions libérales (huit représentants), par le comité de liaison des institutions ordinales (huit représentants). Chacun des trois secteurs d'activité des professions libérales doit être représenté : les professions de santé, les professions juridiques et les professions techniques et du cadre de vie (art. 9 du décret de 2011).

L'exemple de la CAPIL montre que la reconnaissance de représentativité est d'abord une forme de reconnaissance à l'égard de la profession mais également de l'Administration : la représentativité n'est pas une condition posée par le décret de 2011 pour siéger. Elle conditionne cependant de facto, la structuration de la représentation de la profession (infra).

La reconnaissance de représentativité confère une autorité, une légitimité pour peser dans les choix publics. Il peut s'agir de contester un modèle économique, comme l'explique ce fonctionnaire du ministère de l'Agriculture en charge des questions sociales à propos de la demande de reconnaissance de représentativité d'une organisation de petits exploitants agricoles née du rejet d'une certaine conception de la Politique agricole commune :

«Alors, pourquoi ils veulent s'implanter avec les employeurs? Parce que leur credo, c'était de baisser les charges. Leur credo, c'est aussi de contester le monopole de la Mutualité sociale agricole, la MSA. La MSA intervient dans le monde agricole au même titre que la CPAM dans les professions industrielles et commerciales. [Nom de l'organisation professionnelle], à travers un certain nombre de plaintes et de procès pour contester le monopole de la MSA, elle s'est fait débouter régulièrement, mais ça ne l'empêche pas de revenir à l'attaque.»

Cette organisation, d'abord présente au sein de la FNSEA et du CNJA (Centre national des jeunes agriculteurs), souhaitait faire évoluer le syndicalisme agricole de l'intérieur. Sa création en tant qu'organisation «autonome» au début des années 1990 témoigne de cet échec. Être reconnu représentatif prendrait alors tout son sens : ce serait l'assurance de pouvoir faire entendre sa voix au sein de la profession et auprès des pouvoirs publics.

La reconnaissance de représentativité peut tout également être un moyen de peser dans les choix économiques, comme le montre l'exemple suivant tiré lui aussi du secteur de l'agriculture.

La récente création de l'Institut technique et scientifique de l'apiculture et de la pollinisation (ITSAP-Institut de l'abeille), association loi de 1901, proposée par le rapport Saddier ${ }^{(78)}$, témoigne des enjeux qui existent au-delà de la seule création

(78) Saddier M. (2008), Pour une filière apicole durable, Rapport au Premier ministre, p. 15. M. Saddier est député UMP de Haute-Savoie. 
d'une instance consultative aux prérogatives limitées. Dans un contexte de surmortalité des abeilles, multifactorielle selon le rapport Saddier, ou bien liée à l'emploi de pesticides comme le Gaucho ou le Cruiser pour l'UNAF (Union nationale des apiculteurs de France) ou le SNA (Syndicat national d'apiculture), siéger dans une telle instance constitue un réel enjeu pour peser sur les évolutions du secteur, d'autant plus lorsqu'est envisagée la création d'une interprofession apicole. Comprendre tous les enjeux autour de la création de cet institut implique de se replacer dans un contexte d'analyse plus global des rapports entre certaines composantes de la FNSEA. Depuis 1984, le SPMF (Syndicat des producteurs du miel de France), l'UNAF et le SNA formaient la commission apicole de la FNSEA, à laquelle ils adhéraient ${ }^{(79)}$. "Cette commission n'avait aucune véritable activité, mais elle permettait aux trois structures de participer à Bruxelles aux travaux du groupe miel du Copa Cogeca, une structure consultative de la Commission européenne qui a permis une bonne évolution de la directive miel, la mise en place du règlement miel qui a permis de financer en France des programmes de recherche appliquée et aider à la mise en place des aides directes aux apiculteurs, notamment pour l'investissement dans le matériel de transhumance (aides Viniflhor)» (ClÉMENT, 2007). Pour le SPMF, cette commission apicole est une coquille vide. "Nous attendons désormais une gestion "normale" de la filière apicole par la FNSEA. Nous avons constaté une évolution très nette de sa part. Le changement d'homme y est certainement pour beaucoup. La FNSEA a la volonté de développer son groupe apicole et est prête à donner au SPMF la place qui lui revient»( ${ }^{(80)}$. Deux conceptions s'opposent dont l'enjeu est, dans un premier temps, un positionnement au sein de la FNSEA : «L'enjeu du rapprochement, ou plutôt de l'intégration de l'apiculture dans l'agriculture va bien au-delà d'une simple normalisation relationnelle. C'est de la survie de l'abeille et des apiculteurs qu'il est question» ${ }^{(81)}$. Pour le SPMF, la représentation apicole au sein d'une interprofession implique un syndicat unique : «La conclusion s'impose : comme toutes les autres productions marginales (cuisses de grenouilles, escargots, lapins, pigeons, dindes de Noël, tabac, etc.), il n'y a pas de place en apiculture pour plusieurs syndicats concurrents, qui créent

(79) Les développements qui suivent sont tirés du dépouillement des éditoriaux de la revue de l'UNAF, Abeilles et fleurs, rédigés par son président, Henry Clément, de 1998 à janvier 2011 (http://www.unaf-apiculture.info/, onglet «Abeilles et fleurs»). Malgré maintes demandes, il n'a pas été possible d'obtenir un rendez-vous.

(80) Rapport moral de l'AG du SPMF, 5 février 2009, Villeneuve Lez Avignon, p. 3.

(81) Rapport moral de l'AG du SPMF, précité, p. 4. la cacophonie, épuisent les énergies et se neutralisent» ${ }^{(82)}$. Dans le rapport moral de l'Assemblée générale du SPMF (à laquelle assistent un porteparole du ministère de l'Agriculture, M. Saddier, auteur du rapport, et le Président de la commission apicole de la FNSEA), l'exemple est donné du «secteur du cheval qui, au bout de trois ans a dû enterrer son interprofession. Devons-nous préciser pourquoi? Eh bien c'est parce qu'on a intégré dès le départ à égalité de droits, l'activité équestre dite "amateur", et les activités des professionnels du cheval. [...] Que le parlement dise clairement ce que recouvrent précisément les termes amateurs, et professionnels à temps complet ou à temps partiel, en précisant sans ambiguité les droits et les devoirs attachés à chaque catégorie et les choses seront beaucoup plus faciles à construire après ». Ces propos doivent être reliés à l'action en justice intentée par le SPMF contestant la qualité d'organisations syndicales à l'UNAF et au SNA, qui rassemblent des particuliers détenteurs de ruches, et demandant à ce qu'il leur soit interdit de se présenter sous la dénomination de syndicat ou d'union de syndicats à défaut de réunir exclusivement des personnes exerçant habituellement l'activité professionnelle d'apiculteur au sens du droit fiscal. La chambre sociale de la Cour de cassation rejette le pourvoi en se référant au très symbolique article 2 de la Convention $n^{\circ} 87$ de l'Organisation internationale du travail (OIT) relative à la liberté syndicale et à la protection du droit syndical ${ }^{(83)}$ ratifiée par la France. Selon la Haute juridiction, l'article L. 2131-2(84) du Code du travail, «qui suppose l'existence d'activités rémunérées à l'exclusion des activités désintéressées ou philanthropiques, ne distingue pas selon que ces activités sont exercées à titre exclusif, accessoire ou occasionnel, ni selon que les revenus qui en sont tirés constituent un revenu principal ou accessoire de sorte que peuvent constituer un syndicat tous les producteurs de miel et que doit être considéré comme tel tout apiculteur qui commercialise ses produits». En contestant la qualité de syndicat ou d'union de syndicats à l'UNAF et au SNA, le SPMF remet en cause leur capacité et, ce faisant, leur légitimité à représenter les intérêts de la profession.

(82) Extrait du site du SPMF : http://www.beekeeping.com/ spmf/unification.htm. Le SPMF faisait partie de l'UNAF au moment de sa création, en 1946

(83) Cass. Soc. 13 janvier 2009, Syndicat des producteurs du miel de France (SPMF) c/ Union nationale de l'apiculture française (UNAF) et Syndicat national de l'apiculture (SNA) $\mathrm{n}^{\circ}$ 07-17692, $\mathrm{P}+\mathrm{B}+\mathrm{R}$ publié au Bulletin, SSL $\mathrm{n}^{\circ} 1389,2$ mars 2009, note FC et extraits du rapport du Conseiller rapporteur Béraud.

(84) «Les syndicats ou associations professionnels de personnes exerçant la même profession, des métiers similaires ou des métiers connexes concourant à l'établissement de produits déterminés ou la même profession libérale peuvent se constituer librement. Par dérogation à ces dispositions, les particuliers occupant des employés de maison peuvent se grouper en syndicat pour la défense des intérêts qu'ils ont en commun en tant qu'employeur de ces salariés.» 
Début 2009, la FNSEA restructure sa filière apicole et accorde cinq voix au SPMF contre une voix consultative au SNA et à l'UNAF. Cela semble être le prix à payer pour un rapprochement entre le SPMF et la FNSEA qui paraît avoir pesé de tout son poids dans la constitution de l'ITSAP-Institut de l'abeille.

Suivie par d'autres organisations professionnelles comme la Confédération paysanne ou le Syndicat national d'apiculture, l'UNAF conteste l'adoption des statuts de l'ITSAP qui s'est faite sans représentant de l'UNAF, du SNA et de la FNOSAD (Fédération nationale des organisations sanitaires apicoles départementales), ainsi que la possibilité pour certains organismes de l'industrie chimique, comme l'Union des industries de la protection des plantes ou l'UIPP (association des firmes phytosanitaires comme Monsanto, Bayer ou Basf), d'intégrer cette instance. Cette contestation a utilisé le canal parlementaire; plusieurs questions écrites ont en effet été posées par deux sénateurs et un député socialistes au ministre en charge de l'Agriculture $^{(85)}$. Ces prises de position s'accompagnent corrélativement d'un positionnement à rebours de la FNSEA : «Durant des années, la FNSEA s'est totalement désintéressée de l'avenir de l'apiculture... En revanche, elle a continué à protéger les produits phytosanitaires comme Gaucho, Régent ou Cruiser imputant les surmortalités d'abeilles à l'incompétence des apiculteurs et aux seuls problèmes sanitaires... Aujourd'hui, elle tente de reprendre la main et cherche à constituer des groupes régionaux d'apiculteurs plus dociles et plus coopératifs que les représentants de l'UNAF. Dans ce même objectif, elle soutient ardemment le SPMF, syndicat ne représentant plus que 150 adhérents, afin de lui permettre d'apparaître comme un vrai syndicat, ce qu'il n'est plus depuis longtemps...»(86).

Par-delà ces joutes, se joue un positionnement institutionnel au sein de la profession : comme adhérent d'un syndicat agricole majoritaire pour le SPMF (au sein d'une section apicole), comme organisation syndicale pour l'UNAF.

La reconnaissance ou l'absence de reconnaissance de représentativité par l'Administration constitue un enjeu beaucoup plus large en termes, par exemple, de choix économiques et de remise en cause des modes de production. Maintenir la représentativité en l'état permet de pérenniser un modèle d'organisation agricole bâti dans les années 1970 sur une logique productiviste. Ouvrir la porte de la représentativité, au sein de l'interprofession,

(85) Question écrite $\mathrm{n}^{\circ} 12085$ de M. Alain Fauconnier (Aveyron), $J O$, Sénat, 28 février 2010, p. 347; question écrite $\mathrm{n}^{\circ} 12214$ de M${ }^{\text {me }}$ Samia Ghali (Bouches-du-Rhône), JO, Sénat, 25 fév., p. 413; question n ${ }^{\circ} 74851$ de J. Lang (Pas-de-Calais), $J O$ du 30 mars, p. 3513 et $J O$ du 4 mai, p. 4948.

(86) Clément H. (président de l'UNAF) (2010), Abeilles et fleurs, éditorial $\mathrm{n}^{\circ} 713$, février. à des organisations qui véhiculent d'autres valeurs conduit, si ce n'est à une remise en cause de ce modèle traditionnel, du moins à une reconnaissance de la légitimité du débat sur la pertinence de ce modèle. Il n'est pas certain que l'Administration soit prête à défier les forces conservatrices, tant à l'extérieur qu'en son sein.

\section{Reconnaître la représentativité : le travail de l'Administration ${ }^{(87)}$}

Tant du point de vue du droit qu' au niveau de l'analyse des pratiques administratives, la reconnaissance de représentativité délivrée par l'Administration est enserrée dans la procédure d'extension. Les conséquences sont importantes : la représentativité est pensée et mise en œuvre pour et dans le cadre de la négociation collective. S'appuyant sur des prérogatives conférées par le Code du travail, ainsi que sur la jurisprudence du Conseil d'État, les services du ministère du Travail ont élaboré, au fil des dossiers de demande de reconnaissance de représentativité, une doctrine, c'est-à-dire un positionnement institutionnel. Reconnaître une organisation permet de faire vivre le dialogue social mais aussi de déterminer le périmètre de la branche.

\section{Reconnaître la capacité à représenter : "l'animation du dialogue social»"(88)}

Dès 1936, le rôle de l'Administration est, selon les textes, celui d'intermédiaire : le ministre du Travail (ou son représentant) peut intervenir dans le règlement des conflits liés à la négociation d'accords collectifs de travail. Dans sa rédaction contemporaine, le Code du travail prévoit qu'à la demande de l'une des organisations syndicales d'employeurs et de salariés représentatives, ou de sa propre initiative, l'autorité administrative peut provoquer la réunion d'une commission mixte paritaire. Elle est tenue de le faire lorsque deux de ces organisations en font la demande (art. L. 2261-20). À la différence de la commission paritaire, la commission mixte paritaire (CMP) comprend un représentant de l'Administration (d'où le terme «mixte»). L'Administration n'y recourt pas systématiquement, comme en atteste l'analyse de ses pratiques au travers des entretiens réalisés. Selon les chiffres communiqués, plus d'un tiers des conventions et accords collectifs de branche est négocié en CMP.

L'intervention de l'Administration doit alors permettre, pour reprendre une expression citée au moins deux fois lors de l'entretien réalisé avec un haut fonctionnaire du ministère du Travail, de veiller

(87) Pour de plus amples développements, se référer à l'article de J.-P. Le Crom dans ce même numéro.

(88) Circulaire du ministère de l'Alimentation, de l'agriculture et de la pêche et du ministère du Travail (SG/SAFSL/SDTPF/ C2009-1525, DGT/N2009/23) du 21 octobre 2009 sur la négociation collective et les procédures d'extension dans les professions agricoles. 
à la «qualité du dialogue social». Reconnaître la représentativité c'est aussi reconnaître la capacité d'une organisation patronale à représenter, c'est-àdire à accepter de s'engager dans une négociation collective. En effet, dès lors que la représentativité est consubstantielle à la procédure d'extension, la volonté exprimée par une organisation patronale de s'engager dans des négociations ou, à l'inverse, son refus catégorique de négocier pèsent dans le processus de reconnaissance de représentativité. «Est-ce un grain de sable dans la négociation collective ou de l'huile dans les rouages", pour reprendre l'expression utilisée par un haut fonctionnaire du ministère du Travail? Par conséquent, si l'analyse des conditions posées par le Code du travail pour être reconnu représentatif, montre que le critère des effectifs est privilégié, il fait cependant partie d'un «faisceau d'indices» qui seront mobilisés différemment suivant le contexte, comme l'explique ce fonctionnaire de la DGT en charge de l'instruction des dossiers de demande de reconnaissance de représentativité (organisation d'employeurs et de salariés) :

«Ce qui est important, c'est la capacité dont fait montre l'organisation qui demande à être reconnue représentative à s'insérer dans un processus de négociation collective. L'Administration privilégie cet aspect : l'organisation est-elle volontaire pour s'engager dans des négociations, souhaite-t-elle s'engager de manière constructive? [...] Car la représentativité qui est conférée par l'Administration est celle liée à la négociation collective, et seulement celle-là.»

Le président de la commission mixte paritaire, nommé par les services centraux de l'administration du Travail (89), doit être un «facilitateur» de dialogue social, suivant les termes d'un président de commission mixte paritaire. "L'animation $d u$ dialogue social» peut aussi se traduire, au niveau local, par la quête d'un interlocuteur patronal comme dans le secteur des services à la personne où l'Unité territoriale (ex-DDTEFP [Direction départementale du travail, de l'emploi et de la formation professionnelle]) a été saisie par l'Union départementale d'une organisation syndicale de salariés qui a constaté une explosion des recours prud'homaux mettant en cause des entreprises de services à la personne du département :

«Sur le champ des services à la personne, y a des grands réseaux associatifs, par exemple l'ADMR [Aide à domicile en milieu rural], là on a des interlocuteurs, et puis y a toutes les petites entreprises privées, entre guillemets, de services à la personne, et là, l'interlocuteur que l'on a identifié [...] on a un club d'entreprises de services à la personne. On a rencontré sa présidente, on lui a parlé du problème. [...] On va essayer de se retourner vers la partie patronale, notamment vers ce club pour leur proposer

(89) Il existe un «vivier» composé, notamment, d'inspecteurs du travail. de participer à une rencontre avec les organisations syndicales, tout en sachant qu'une rencontre ça peut s'organiser y compris avec des gens pas représentatifs mais que si on veut arriver à une négociation, il faudra que d'une manière ou d'une autre, ils se constituent. Alors ils adhèrent à l'UPE [Union patronale des employeurs] ou ils adhèrent directement au Medef, ou ils adhèrent à l'organisation patronale de branche qui s'est constituée au niveau national.»

(Un fonctionnaire d'une Unité territoriale.)

La quête de la représentation patronale peut aider à structurer l'activité économique locale et les conditions de travail, comme l'explique un fonctionnaire d'une Unité territoriale :

«Moi ce qui m'importe, c'est que j'aie en face de moi un syndicat représentatif qui peut signer. Je vous donne un autre exemple : y a très longtemps, dans un registre différent, sur St Malo. En février 2002, un inspecteur et un contrôleur du travail font des contrôles le dimanche dans St Malo intra-muros et ils constatent que tous les commerces sont ouverts et il y a eu des plaintes de la part des salariés. Ils menacent de dresser un procès-verbal. L'association des commerçants de l'intra-muros s'en émeut, alerte le sous-préfet qui alerte le préfet et le préfet m'alerte. Qu'est-ce qu'on fait? On a dit : et bien écoutez, on va vous montrer le constat et puis on va tout autour d'une table les uns et les autres. On a mis autour d'une table les cinq syndicats ouvriers et on a mis autour de la table l'association des commerçants de l'intra-muros. Mais rapidement on s'est posé la question de sa représentativité. Je leur ai dit : y a pas d'autre solution, il faut adhérer à l'UDE [Union des entreprises] 35. Ils ont adhéré à l'UDE 35 qui adhère au Medef; ils étaient représentatifs et on a réussi à passer des accords. On a passé trois accords, sur le travail du dimanche dans St Malo, et aujourd'hui les salariés de l'intra-muros, uniquement l'intra-muros, travaillent... euh... vingt-trois dimanches par an ou vingt-quatre dimanches par an et sont rémunérés à $100 \%$. Auparavant ils travaillaient tout le dimanche et payés zéro, enfin, pas de majorations. »

La commission mixte paritaire de branche constitue un observatoire des relations professionnelles et la reconnaissance (ou le refus de reconnaissance de représentativité) peut aussi être utilisée comme un moyen de favoriser les évolutions ou un certain statu quo du secteur d'activité. Reconnaître ou exclure de la représentativité certaines organisations patronales contribue à redessiner les relations professionnelles d'un secteur d'activité et l'Administration préfère parfois maintenir en place une organisation «historique» qui, bien qu'étant peu représentative du secteur d'activité, porte cependant la mémoire de l'histoire de la branche face à deux «nouveaux entrants» qui n'ont que quelques mois d'ancienneté et très peu d'expérience en tant que négociateurs. La décision juridique de maintien de la représentativité donnée par le ministère du Travail s'appuie alors sur une «géopolitique» de la branche. 
Par ailleurs, reconnaître représentative une organisation patronale c'est aussi reconnaître sa capacité à relayer une politique publique de l'emploi, par exemple. L'Administration peut aussi chercher à favoriser le pluralisme syndical patronal comme moyen de nourrir le dialogue social ou, plus prosaïquement, éviter une position hégémonique d'une organisation majoritaire. De ce point de vue, la reconnaissance de représentativité véhicule une dimension politique incontestable. Comme l'explique très clairement ce fonctionnaire de la DGT, «l'extension n'est pas une mécanique», elle implique de tenir compte du cadre dans lequel est faite la négociation. S'appuyant sur des textes interprétés par le Conseil d'État, ce paritarisme est orienté par l'État.

\section{Déterminer le périmètre de la branche et éviter les chevauchements}

En 1936 apparaît, dans le droit, la notion de branche d'activité. Elle est intimement liée à sa délimitation, ce que l'on appelle aujourd'hui, en termes plus contemporains, son «périmètre».

Comment déterminer les contours de la branche? Ce travail incombe-t-il aux partenaires sociaux ou à l'Administration? Faut-il laisser le soin à chaque accord de déterminer son champ d'application ou devrait-il y avoir une délimitation juridique $a$ priori de la notion de branche?

Au regard de la loi du 25 juin 1936, les branches d'activité sont d'abord des branches industrielles qui possèdent des caractéristiques propres : «l'existence d'une branche d'activité était admise chaque fois qu'une activité présentait des caractères originaux en raison de la matière première mise en œuvre, de l'organisation technique du travail, des classifications du personnel, de la structure donnée par les intéressés à leurs organisations professionnelles » (Arseguel, 1976, p. 382). Aucune loi ne définit la branche. La détermination de ses contours est liée au champ d'application de la convention et donc à l'accord de volonté des signataires. C'est au gré des contentieux sur la légalité de l'arrêté d'extension et de la contestation, par des non-signataires, de la représentativité de l'organisation patronale signataire que se redessine le périmètre des branches. Bien que dénuées de valeur juridique ${ }^{(90)}$, les nomenclatures des activités économiques dressées par l'Insee jouent un rôle important.

Plusieurs arrêts rendus par le Conseil d'État dans les années 1960 dans des affaires qui concernaient la branche des industries chimiques contribuent à l'émergence d'une certaine approche de la notion en même temps qu'ils tracent les contours de l'intervention de l'Administration. Dans la décision du

(90) Cass. soc. 4 mars 1964, Bull. IV, n 202; Cass. Soc. 22 octobre $2008, n^{\circ} 07-42193$, non publié au bulletin.
4 mars 1960, Fédération nationale des industries chimiques et parties similaires, la branche est, pour le commissaire du gouvernement Nicolaÿ (dont le rôle sera déterminant dans la formation d'une doctrine jurisprudentielle), une notion qu'il appartient au ministre du Travail, sous le contrôle du juge administratif, de définir dans le cadre de la procédure d'extension. Car s'il incombe bien aux partenaires sociaux de définir le champ d'application de l'accord de branche, c'est ensuite à l'Administration qu'il revient, lors de l'extension, de vérifier le périmètre de la branche. Ce faisant, le ministre évite ainsi les «chevauchements» de branches ${ }^{(91)}$.

Deux arrêts du 2 mars $19622^{(92)}$ concernant la même branche des industries chimiques, rendus là encore sous les conclusions du commissaire du gouvernement Nicolaÿ, confortent cette doctrine jurisprudentielle. Nicolaÿ propose alors que le ministre du Travail établisse, lors de son contrôle, une distinction entre la «branche d'activité au cadre étroit» - «c'est une branche d'activité qui constitue un tout homogène $[\ldots]$ au sein de laquelle on ne conçoit pas de sécessions» - et les "groupements de branches d'activités» qui réunissent un certain nombre de branches d'activités «qui s'apparentent entre elles par une unité de nature d'activité»(93). Pour le commissaire du gouvernement, les mesures d'extension peuvent concerner plusieurs branches d'activité dès lors qu'elles rassemblent des activités de même nature, et à condition que les organisations représentatives aient adhéré à la convention. Autrement dit, l'appréciation de la validité de l'accord susceptible d'extension dépendrait de l'acception retenue du périmètre de la branche. Semble se profiler la notion d'interbranche / multibranche, l'un des enjeux actuels du droit de la négociation collective ${ }^{(94)}$.

Mais la distinction ainsi faite entre la branche d'activité au sens strict du terme et les «groupements de branches» est fortement dépendante de la structuration de la branche et donc de l'activité économique et de la représentation patronale. Les prérogatives de l'Administration se trouvent considérablement élargies par une telle interprétation. Elles sont «conformes à l'esprit de la législation dont il faut bien reconnaître qu'elle l'a invité beaucoup moins à dresser de façon dogmatique la carte des corporations qu'à apporter de manière empirique aux découpages opérés par les professions les

(91) Depuis la loi du 4 mai 2004, les partenaires sociaux peuvent insérer des clauses de «répartition» visant à éviter ces chevauchements.

(92) «CE 2 mars 1962, Fédération nationale des industries chimiques et Union nationale des chambres syndicales de miroitiers (deux arrêts) rendus sur les conclusions du commissaire du gouvernement Nicolaÿ», Droit social, 1962, $\mathrm{n}^{\circ} 6$, p. 346 et ${ }^{\circ} 12$, p. 604.

(93) Ibid., pp. 351-352.

(94) Pour plus de développements sur ce point, $c f$. l'article de Pascal Caillaud dans ce numéro. 
retouches que peut imposer un arbitrage uniquement soucieux d'éviter usurpations ou doubles appartenances » (95). L'interprétation constructive du Conseil d'État et, plus spécialement de son commissaire du gouvernement, confère à l'extension un effet structurant sur la branche d'activité. Cet usage de l'extension a été récemment consacré par un arrêt du Conseil d'État du 23 juillet 2010 (96) qui reconnaît la validité d'un arrêté ayant pour seule fonction de fixer le périmètre d'un accord de branche. L'accord national professionnel du 27 avril 2007 signé, du côté patronal, par le SYNESI(97) et étendu par l'arrêté d'extension du 10 décembre 2007 a pour objet exclusif de fixer le champ d'application des futurs accords collectifs relatifs aux employeurs et salariés de droit privé des ateliers et chantiers d'insertion. L'arrêté d'extension est attaqué par l'Unifed (Union des fédérations et syndicats nationaux d'employeurs sans but lucratif du secteur sanitaire, médico-social et social) qui estime que le champ d'application ainsi défini empiète pour partie sur celui des accords de la branche sanitaire, sociale et médico-sociale à but non lucratif. Par une interprétation extensive de l'article L. 2261-15(98), le Conseil d'État reconnaît une telle prérogative au ministre chargé du Travail, alors même que l'accord en question ne comporte aucune stipulation de fond. Suivant le commissaire du gouvernement Anne Courrèges, l'arrêté d'extension vient consolider une première étape qui commence par la discussion du champ de la future convention.

«Même si, en apparence, il n'étend que du "vide", il permet de figer un élément clef de la négociation le périmètre professionnel -, ce qui a au moins deux effets immédiats : d'une part, définir en conséquence la liste des organisations d'employeurs et de salariés représentatives dans le champ d'application considéré et donc appelées à négocier et conclure la future convention de branche ou l'accord professionnel ou interprofessionnel, conformément à l'article L. 133-1 du code de travail, devenu l'article L. 2261-19;

(95) Conclusion du commissaire du gouvernement Nicolaÿ précitée, p. 352.

(96) CE 23 juillet 2010, n 313 776, cf. Champeaux F. (2010), "La légalité de l'extension d'un accord "vide"», Semaine sociale Lamy, 6 sept., $\mathrm{n}^{\circ} 1457$, pp. 12-13 et Courrèges A. (2010), «Légalité de l'extension d'un simple accord de champ, sans stipulation de fond. Concl. sous Conseil d'État, 23 juillet $2010 »$, Droit social, n $^{\circ}$ 693, novembre, pp. 1085-1091.

(97) Syndicat national des employeurs spécifiques d'insertion, créé le 19 juin 2006 par quatre réseaux du secteur de l'insertion économique (Chantier école, Cocagne, Coorace et Tissons la solidarité). Il est adhérent à l'Usgeres depuis janvier 2008 (http://www.synesi.fr/).

(98) «Les stipulations d'une convention de branche ou d'un accord professionnel ou interprofessionnel, répondant aux conditions particulières déterminées par la sous-section 2, peuvent être rendues obligatoires pour tous les salariés et employeurs compris dans le champ d'application de cette convention ou de cet accord, par arrêté du ministre chargé du travail, après avis motivé de la Commission nationale de la négociation collective. L'extension des effets et des sanctions de la convention ou de l'accord se fait pour la durée et aux conditions prévues par la convention ou l'accord en cause». d'autre part, donner au ministre un fondement légal solide pour empêcher les chevauchements de champ, notamment pour protéger les négociations en cours d'une "captation" par d'autres conventions" (Courrèges, 2010, p. 1087).

L'Administration, appuyée en cela par une jurisprudence bienveillante du Conseil d'État, se voit reconnaître plus qu'une prérogative, un véritable pouvoir de réorganiser les branches professionnelles.

«La définition du champ d'application conventionnel est en effet un enjeu pratique majeur. L'importance de la question a encore gagné en acuité du fait du travail actuel de recomposition des branches, mais aussi, depuis la loi du 20 août 2008, à raison des adhérences avec les questions de représentativité. Dans ce cadre, la démarche d'extension d'accords "de champ" ne constitue pas une initiative isolée. Elle s'inscrit dans le cadre d'un double mouvement d'ensemble, l'un, ancien, visant à favoriser le développement de la négociation conventionnelle, et l'autre, plus récent, de recomposition des branches vers des ensembles moins nombreux mais plus cohérents et actualisés. Cette démarche a d'ores et déjà concerné de nombreux secteurs, souvent sensibles. Le cas des services d'aide à la personne le montre bien; on peut aussi citer l'opération ayant conduit à passer de 40 à 8 branches dans le secteur du spectacle et de l'audiovisuel ou l'"autonomisation" de la branche du sport ou encore la négociation de la convention collective des sites d'hôtels-restaurants-thalassothérapie. » (COURRÈGES, 2010, p. 1088.)

Pour bien comprendre la portée de cette décision, il est important de la mettre en perspective avec la volonté des services du ministère du Travail de réorganiser les branches professionnelles afin d'en réduire le nombre, estimé par ses services à environ 687 «connues de la DGT»(99), dont 162 relevant du secteur dit «général» de plus de 5000 salariés (près de 400 branches regroupent moins de 5000 salariés). Sur ces 687 branches, près de la moitié a signé au moins un accord salarial sur une période de trois ans. La DGT souhaite procéder à des regroupements de branches qui tiennent compte de la similitude des activités (refus de prendre en compte le statut des entreprises), du nombre de salariés (au moins 10 000) et de l'activité conventionnelle. Cette doctrine ministérielle est relayée par les pratiques administratives, comme cela ressort très clairement de cet extrait d'entretien réalisé avec un fonctionnaire de la DGT en charge de l'instruction des dossiers de demande de reconnaissance de représentativité :

«On essaye au maximum de ne pas reconnaître des sous-secteurs dans une convention collective.

- Pourquoi?

(99) Chiffres cités par le rapport Poisson : Poisson J.-F (2009), Rapport sur la négociation collective et les branches professionnelles, La Documentation française, $528 \mathrm{p}$. 
- Dans ce cas-là, on se retrouverait avec des conventions collectives où il y aurait dix syndicats. On est dans une logique où on est plus dans une fusion des champs d'application. On a des conventions collectives qui regroupent des activités très diverses et si on imagine que, pour chaque activité, il y a un syndicat, on ne va jamais s'en sortir. Parce qu'après, il faut voir les conséquences. Nous, quand on reconnaît une organisation sur un champ donné, ça veut dire qu'au moment de l'extension quand cette organisation a signé un accord, si elle signe toute seule, l'accord est quand même étendu à l'ensemble des salariés de ce champ. Or, si c'est une organisation sectorielle, ça ne sera qu'au morceau qu'elle représente. Alors, là, ça fait un bazar, ça morcelle complètement la négociation. 》

Par ailleurs, les prérogatives du ministre du Travail s'exercent également sur le contenu de la négociation collective. Si le ministre chargé du Travail est tenu d'engager la procédure d'extension (art. L. 2261-24, 2e al.), il n'est pas tenu d'étendre le texte. Il peut, après avis motivé de la Commission nationale de la négociation collective, exclure certaines clauses de l'extension ${ }^{(100)}$. Les pouvoirs du ministre en ce domaine sont vastes : l'article L. 2261-25 lui confère un «[...] pouvoir d'appréciation lui permettant de refuser cette extension, sous le contrôle du juge de l'excès de pouvoir, pour des motifs d'intérêt général tenant notamment aux objectifs de la politique économique et sociale ou à la protection de la situation des tiers $\gg(101)$. Il peut s'agir, par exemple, de favoriser le maintien dans l'emploi des seniors, conformément au plan d'action que le Gouvernement avait annoncé le 6 juin 2006. Le contrôle exercé par le Conseil d'État est un contrôle restreint, comme l'explique le commissaire du gouvernement Anne Courrèges dans ses conclusions : «nous avouons quelque gêne à l'idée que le choix d'étendre un accord collectif de travail ne se prête pas au minimum à un contrôle restreint alors qu'il conduit à une immixtion des pouvoirs publics dans le libre jeu de la négociation collective, régi par le principe d'autonomie des partenaires sociaux » (CourrèGes, 2009, p. 9). C'est lors de l'extension que le ministre du Travail doit, selon le Conseil d'État, «rechercher si le champ d'application professionnel défini en termes d'activités économiques pour lequel l'extension est envisagée n'est pas compris dans le champ professionnel

(100) Art. L. 2261-25 : «Le ministre chargé du travail peut exclure de l'extension, après avis motivé de la Commission nationale de la négociation collective, les clauses qui seraient en contradiction avec des dispositions légales. Il peut également exclure les clauses pouvant être distraites de la convention ou de l'accord sans en modifier l'économie, mais ne répondant pas à la situation de la branche ou des branches dans le champ d'application considéré. Il peut, dans les mêmes conditions, étendre, sous réserve de l'application des dispositions légales, les clauses incomplètes au regard de ces dispositions.»

(101) Conseil d'État 21 novembre 2008, n 300 135. Publié au Recueil Lebon; concl. du commissaire du gouvernement A. Courrèges. et territorial d'une autre convention ou d'un autre accord collectif étendu par arrêté». Le cas échéant, il appartient au ministre, préalablement à l'extension projetée, «soit d'exclure du champ de l'extension envisagée les activités économiques déjà couvertes par la convention ou l'accord collectif précédemment étendu, soit d'abroger l'arrêté d'extension de cette convention ou de cet accord collectif en tant qu'il s'applique à ces activités dans le secteur territorial considéré»» ${ }^{(102)}$.

Comme le montrent les exemples donnés, la reconnaissance de représentativité véhicule de multiples enjeux : maintenir le statu quo ou ouvrir la représentation n'est pas sans incidence sur la pérennisation ou l'évolution des relations sociales. Mesurer l'audience des organisations patronales peut amener une recomposition du paysage syndical. Comme l'explique ce fonctionnaire du ministère de l'Agriculture en charge des questions sociales dans cet extrait d'entretien :

«Si on disait que le Medefn'est pas représentatif, cela ferait complètement tomber le système du dialogue social en France. Personne, et en premier lieu les syndicats de salariés, personne n'a réellement intérêt à ce qu'on aille trop regarder la représentativité réelle des uns et des autres».

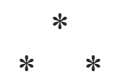

Née des rapports de travail, la représentativité couvre aujourd'hui un champ plus vaste et véhicule d'autres enjeux. Ancrée, d'un point de vue historique, dans la procédure d'extension des conventions et accords collectifs de travail, elle permet de siéger dans diverses instances nationales consultatives comme le Conseil national de la consommation ou la Commission des clauses abusives et ouvre également des droits à percevoir des financements au titre du paritarisme. Si les fonctions de la représentativité se sont progressivement élargies - 1'institutionnalisation du paritarisme de branche ou national et interprofessionnel suppose la mise en place de procédures de sélection des organismes appelés à siéger - l'utilisation qui en est faite par les organisations reconnues représentatives (ou qui demandent à l'être) ou encore par l'Administration, montre à quel point ces enjeux dépassent le seul cadre juridique. Conférée par une autorité tierce, la représentativité constitue une forme de reconnaissance et de légitimation.

Si un projet ou une proposition de loi établissant des critères de mesure de la représentativité des organisations professionnelles d'employeurs devait être déposé, cela impliquerait nécessairement de

(102) CE 15 mai 2006, nº 270 174, mentionné dans les tables du Recueil Lebon 
poser au préalable la question de ses finalités. Les critères de reconnaissance de la capacité juridique d'une organisation professionnelle d'employeurs doivent-ils être identiques alors même que les fonctions attachées à la reconnaissance de représentativité divergent, suivant qu'il s'agit de siéger dans des instances paritaires ou de signer des conventions et accords susceptibles d'être étendus?

Par ailleurs, une telle réforme devrait nécessairement poser la question de la place de l'administration du Travail dans la structuration des relations socioprofessionnelles. Outil d'uniformisation des conditions de Travail limitant les distorsions de concurrence par un abaissement des coûts sociaux, le mécanisme de l'extension est devenu, au fil des réformes, un levier de la politique sociale de l'État. Cet interventionnisme utilise deux canaux : les incitations à la négociation collective (négociations sur l'emploi des seniors, la pénibilité, par exemple, au niveau national et interprofessionnel) et les observations «constructives» émises par les services du ministère du Travail lors de la procédure d'extension qui peuvent conduire à une extension sous réserve de renégociation de certaines clauses de la convention ou de l'accord collectif de travail. Plus largement, les relations sociales en France accordent un rôle tout particulier à l'État dont l'intervention prend de multiples formes allant de la gestion tripartite (103) à l'association des partenaires sociaux à l'élaboration de la loi négociée(104) (le législateur n'étant cependant pas lié par l'accord national interprofessionnel).

Parachever la réforme de la représentativité en l'étendant aux organisations professionnelles d'employeurs devrait conforter leur légitimité et asseoir leur autorité, permettant, peut-être, d'opposer à cet interventionnisme étatique des principes juridiques en devenir dans le champ des relations sociales comme l'autonomie des partenaires sociaux et la liberté contractuelle.
(103) L'exemple peut être donné du secteur de la formation professionnelle continue. La signature de conventions cadres signées entre le Fonds paritaire de sécurisation des parcours professionnels, organisme paritaire créé par l'ANI du 7 janvier 2009 , et l'État ou encore la signature de COM, conventions d'objectifs et de moyens chargées de définir les modalités de financement et de mise en œuvre des missions des organismes collecteurs paritaires agréés (art. L. 6332-1-1 cod. trav.) s'apparentent à une forme de tutelle de l'État sur les organismes paritaires.

(104) La loi du 31 janvier 2007 dite de modernisation du dialogue social (cod. trav. art. L. 1 à 3) prévoit que tout projet de réforme envisagé par le Gouvernement qui porte sur les relations individuelles et collectives du travail, l'emploi et la formation professionnelle doit être précédé d'une concertation préalable avec les organisations syndicales de salariés et d'employeurs représentatives au niveau national et interprofessionnel qui peuvent décider d'ouvrir des négociations. 


\section{Bibliographie}

Arseguel A. (1976), La notion d'organisations syndicales les plus représentatives, Thèse de droit, école doctorale sciences juridiques et politiques, Université de Toulouse 1, 2 tomes, $698 \mathrm{p}$.

BONNARD R. (1937), «Syndicalisme, corporatisme et État corporatif», Revue du droit public, pp. 58-123.

Borenfreund G. (2008), "Le nouveau régime de la représentativité syndicale», Revue de droit du travail, $\mathrm{n}^{\circ} 12$, décembre, pp. 712-722.

Colson F. (2008), «Que reste-t-il de la cogestion Étatprofession?», in Ayats J.-F., Cordellier S., Vincent J., Voisin L.-M. (coord.), «L'univers des organisations professionnelles agricoles », Pour, n 196-197, pp. 107-113.

Courrèges A. (2009), «Le pouvoir d'appréciation du ministre pour refuser en opportunité l'extension. Conclusion sous l'arrêt CE 21 nov. 2008, n 300135 », Semaine sociale Lamy, ${ }^{\circ}$ 1384, 26 janvier, pp. 6-10.

Courrèges A. (2010), «Légalité de l'extension d'un simple accord de champ, sans stipulation de fond. Concl. sous Conseil d'État, 23 juillet 2010», Droit social, $n^{\circ}$ 693, novembre, pp. 1085-1091.

DuRAND P. (1939), «Le dualisme de la convention collective», Revue trimestrielle de droit civil, pp. 353-392.
HÉAS F. (2011), «La représentativité des organisations salariées et patronales en droit français », Revue de droit du travail, $\mathrm{n}^{\circ} 2$, pp. 91-98.

LAFORE R. (2001), «À propos de la convention du 1 er janvier 2001 : où en est le paritarisme?», Droit social, avril, pp. 347-354.

LAROQUE P. (1934), Les conventions collectives de travail, Paris, Imprimerie nationale.

Maggi-Germain N. (2009) (dir. scientifique), Construire l'insertion des travailleurs handicapés : le rôle de la négociation collective, 304 p. Rapport téléchargeable sur HAL-SHS (Hyper Article en Ligne - Sciences de l'Homme et de la Société) : http://halshs.archivesouvertes.fr/halshs-00411532/fr/

Maggi-Germain N. (2011), «La représentativité des organisations professionnelles d'employeurs», Droit social, $\mathrm{n}^{\circ} 11$, novembre, pp. 1072-1082.

Pollet G., Renard D. (1995), «Genèses et usages de l'idée paritaire dans le système de protection sociale français, fin $19^{\mathrm{e}}$ - milieu du $20^{\mathrm{e}}$ siècle $»$, Revue française de science politique, vol. 45, $\mathrm{n}^{\circ} 4$, pp. 545-569.

Rouast A. (1909), Essai sur la notion juridique de contrat collectif dans le droit des obligations, Thèse, Paris, A. Rousseau, 434 p. 